\title{
Synthesis of Menthol Glycinates and Their Potential as Cooling Agents
}

Douglas A. Klumpp*,a, Robert M. Sobelc, Smaro G. Kokkinidouc, Brian Osei-Badu a , Zachary Liveris $^{a}$, Rachel A. Klumpp ${ }^{b}$, and Michael R. Stentzel ${ }^{a}$

aDepartment of Chemistry and Biochemistry

Northern Illinois University

DeKalb, IL 60178

b. Aurora Christian Schools

2255 Sullivan Road

Aurora, IL 60506

cFONA International, Inc.

1900 Averill Rd

Geneva, IL 60134

$\begin{array}{ll}\text { Pages } & \text { Content } \\ \text { S1 } & \text { Index } \\ \text { S2-S19 } & { }^{1} \mathrm{H} \text { and }{ }^{13} \mathrm{C} \text { NMR spectra of compounds }\end{array}$




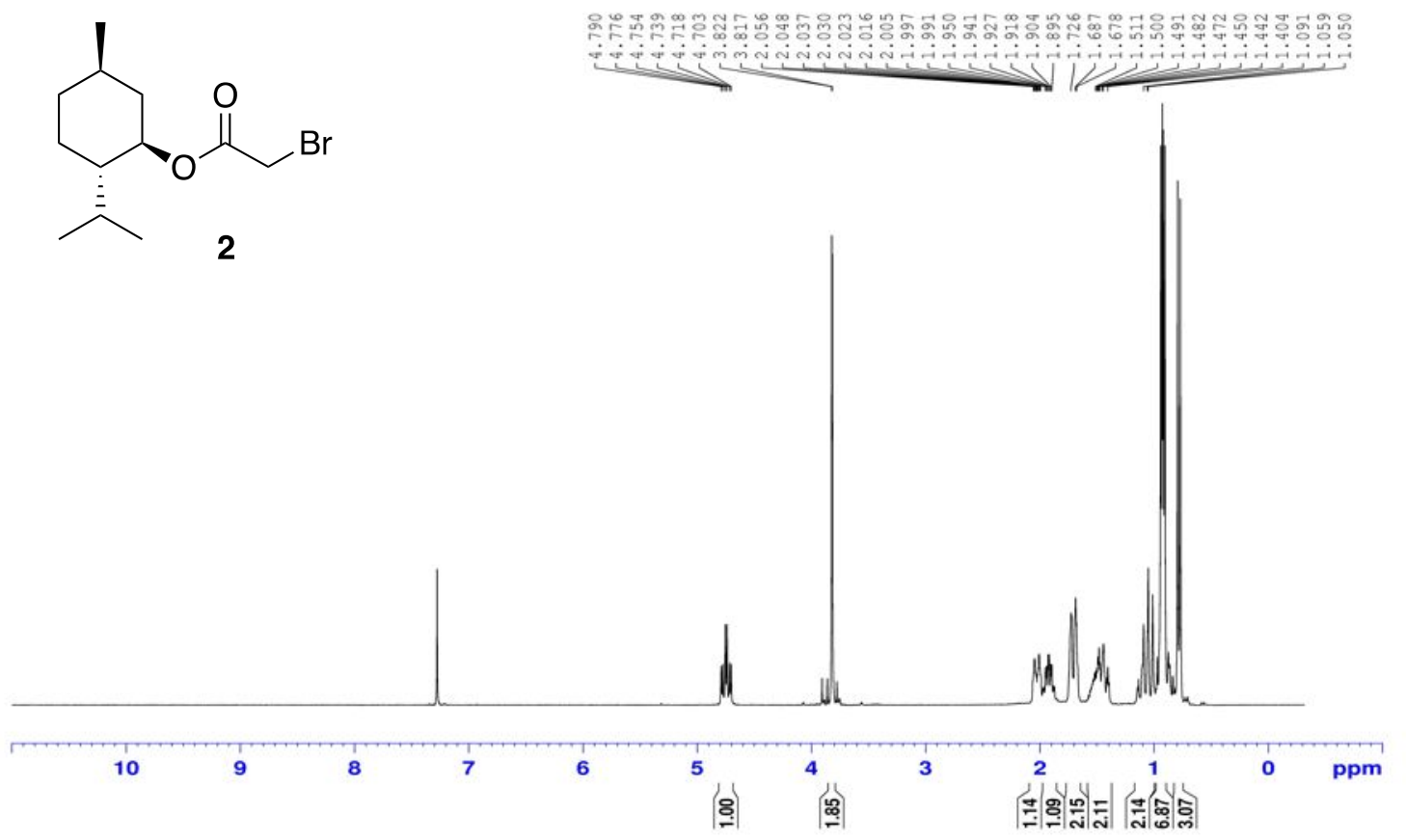

Figure S1. 1H NMR of compound $2\left(\mathrm{CDCl}_{3}, 300 \mathrm{MHz}\right)$.

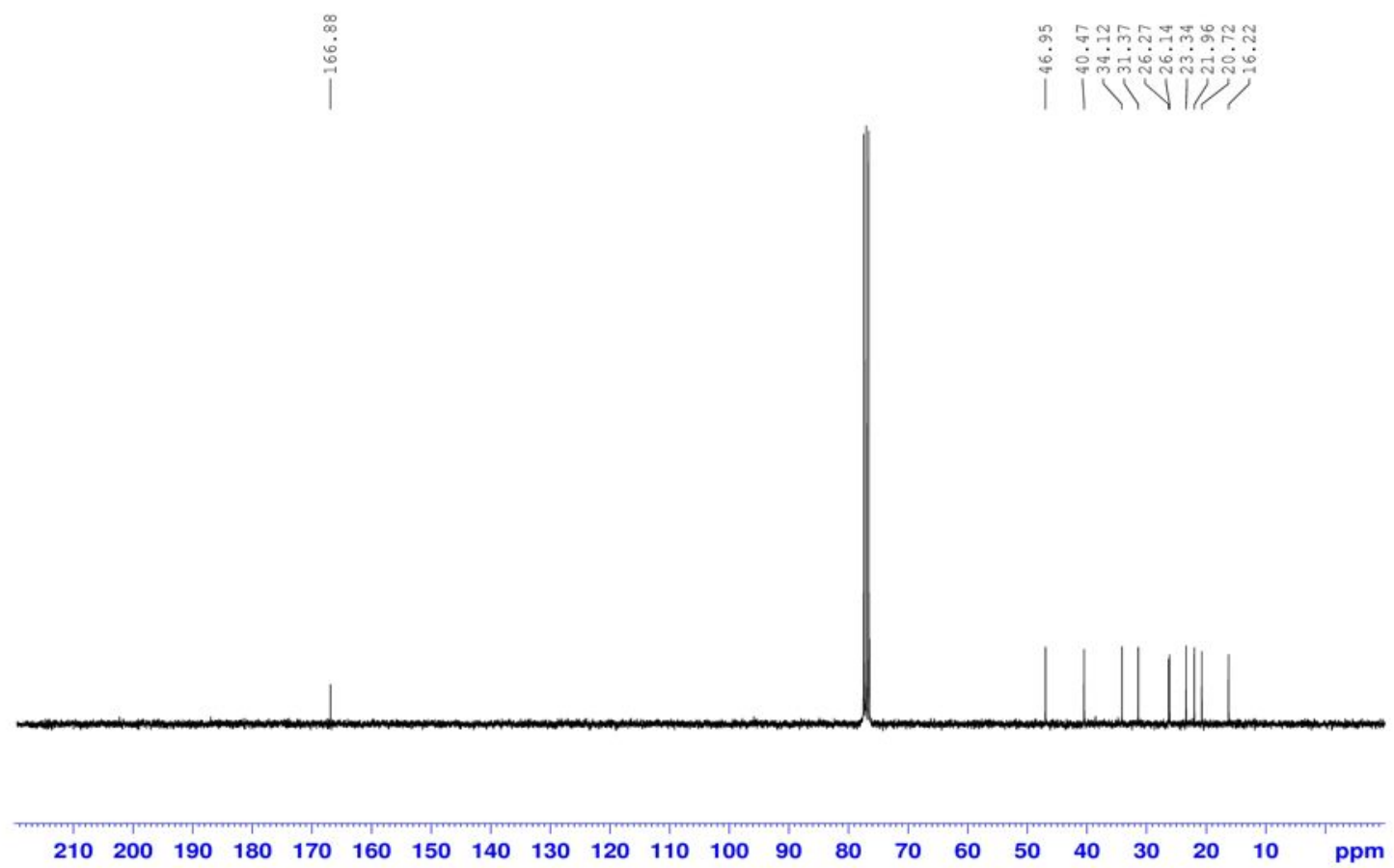

Figure S2. $13 \mathrm{C} \mathrm{NMR} \mathrm{of} \mathrm{compound} 2\left(\mathrm{CDCl}_{3}, 75 \mathrm{MHz}\right)$. 

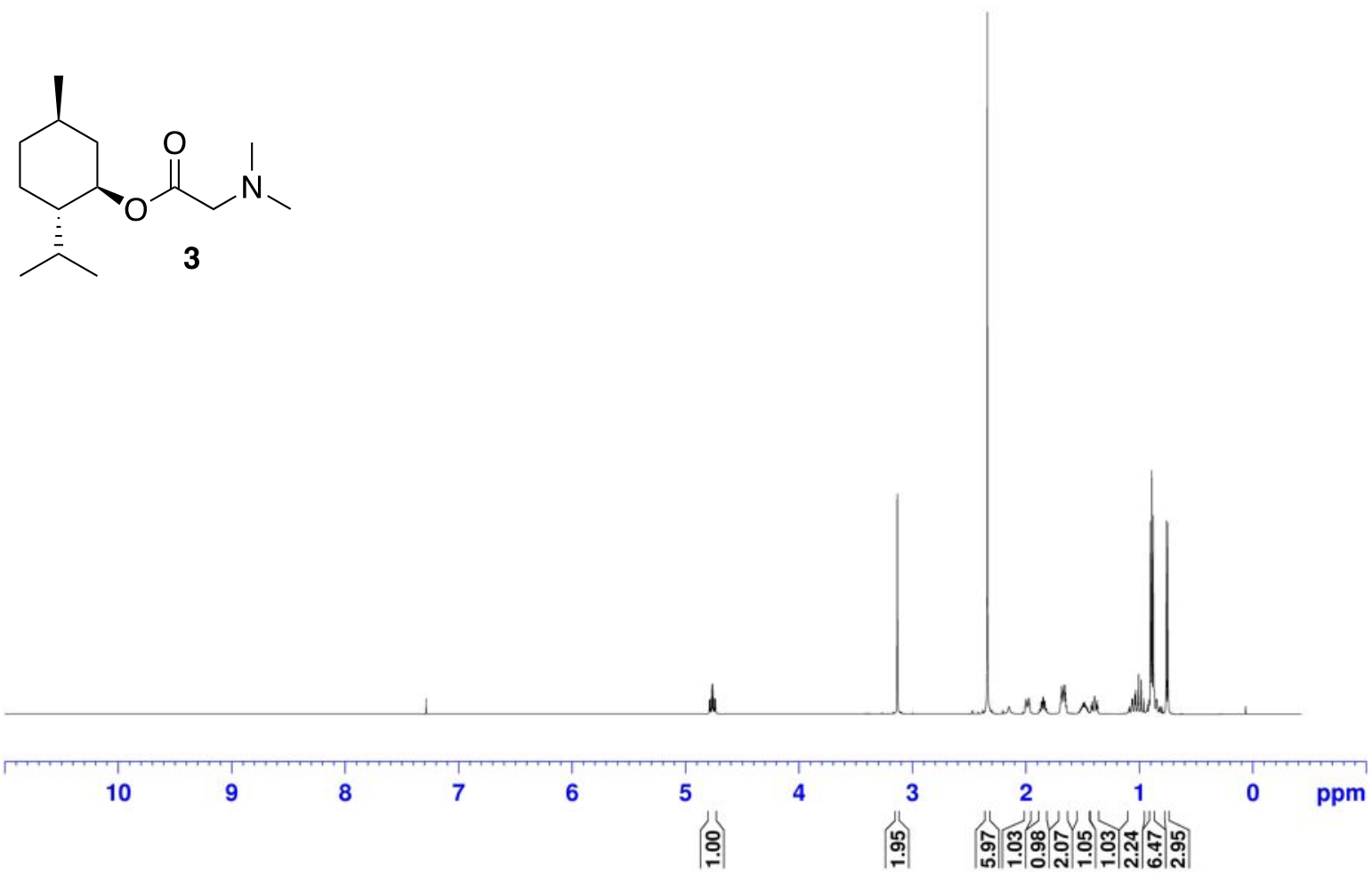

Figure S3. $1 \mathrm{H} \mathrm{NMR}$ of compound $\mathbf{3}\left(\mathrm{CDCl}_{3}, 300 \mathrm{MHz}\right)$.

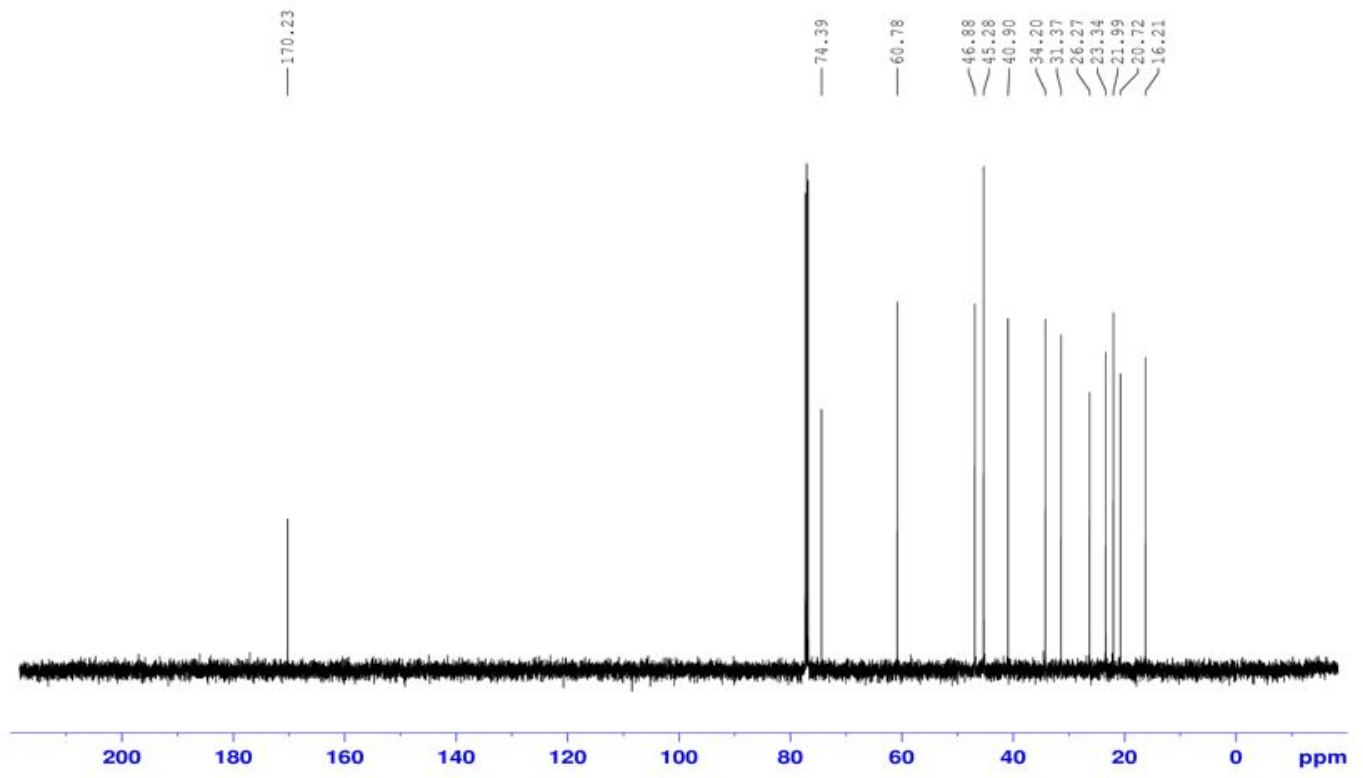

Figure S4. $13 \mathrm{C}$ NMR of compound $3\left(\mathrm{CDCl}_{3}, 75 \mathrm{MHz}\right)$. 


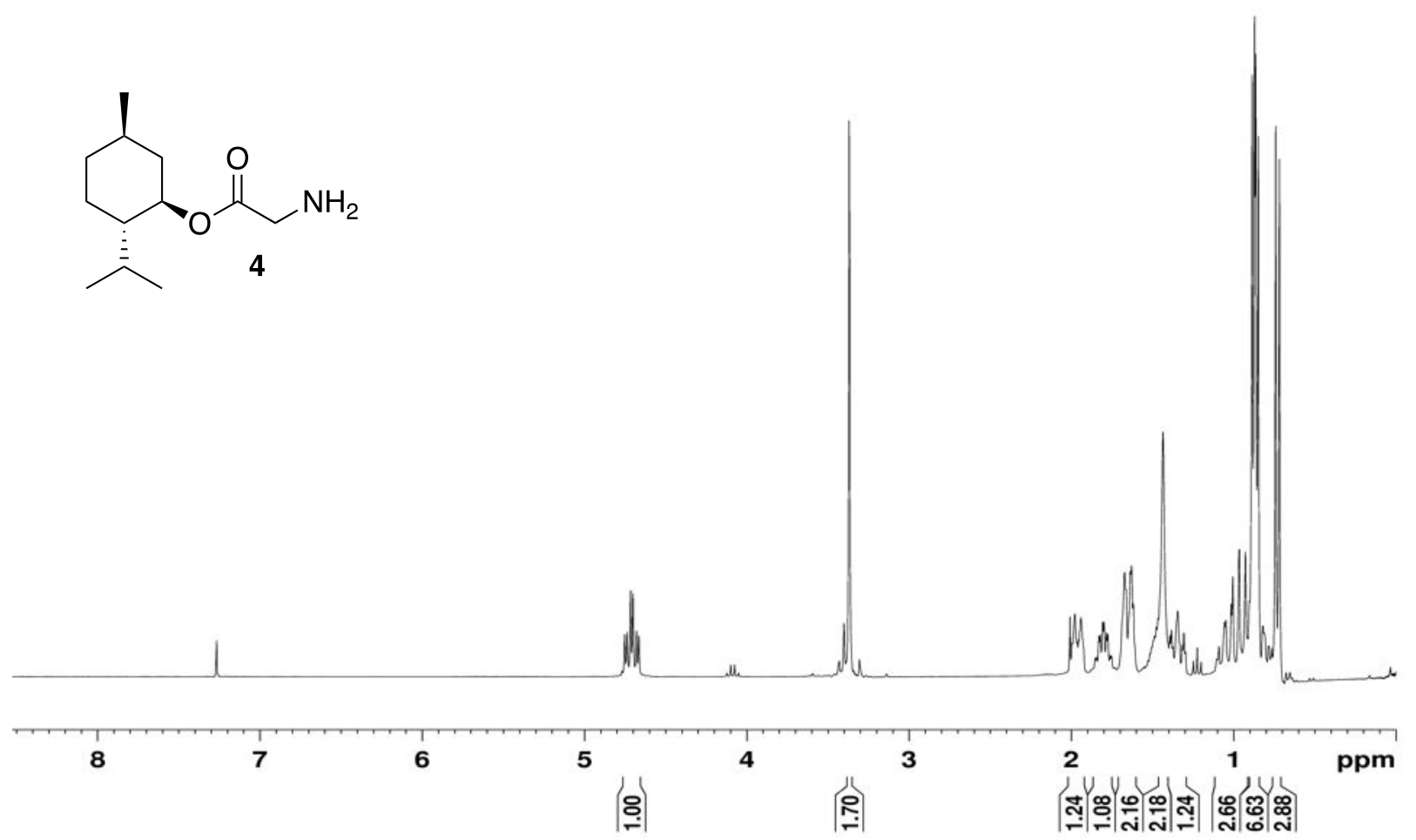

Figure S5. $1 \mathrm{H} \mathrm{NMR}$ of compound $4\left(\mathrm{CDCl}_{3}, 300 \mathrm{MHz}\right)$.

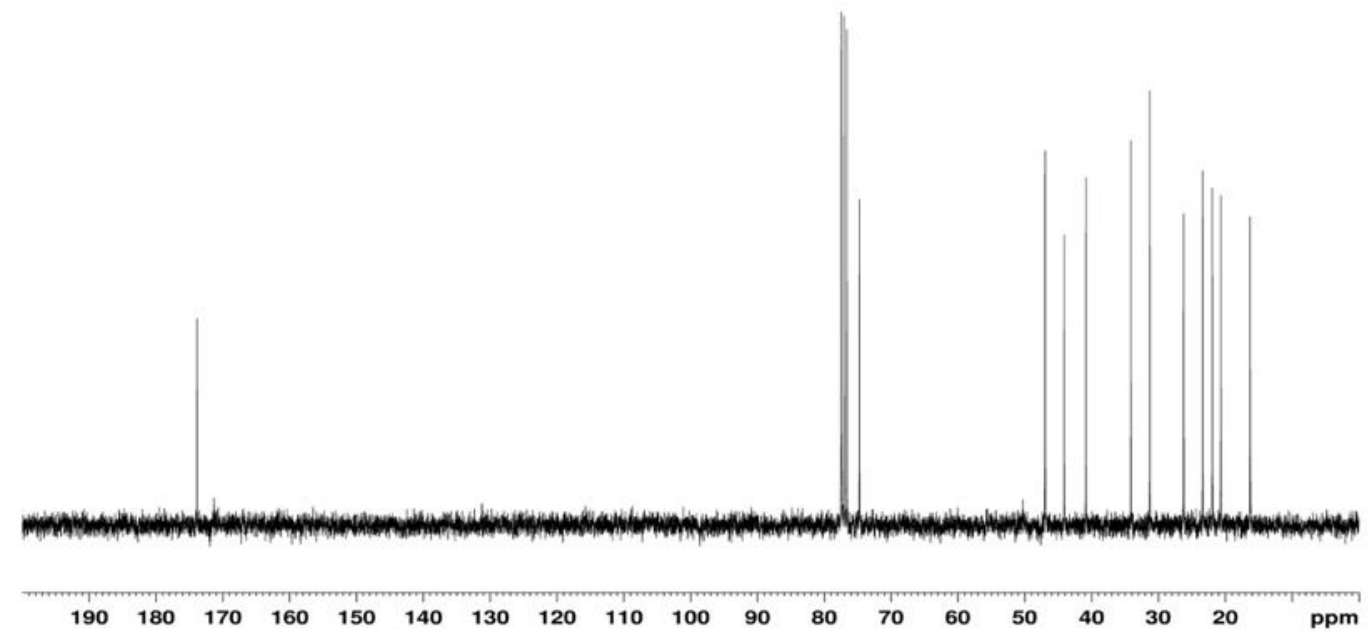

Figure S6. $13 \mathrm{C}$ NMR of compound $4\left(\mathrm{CDCl}_{3}, 75 \mathrm{MHz}\right)$. 


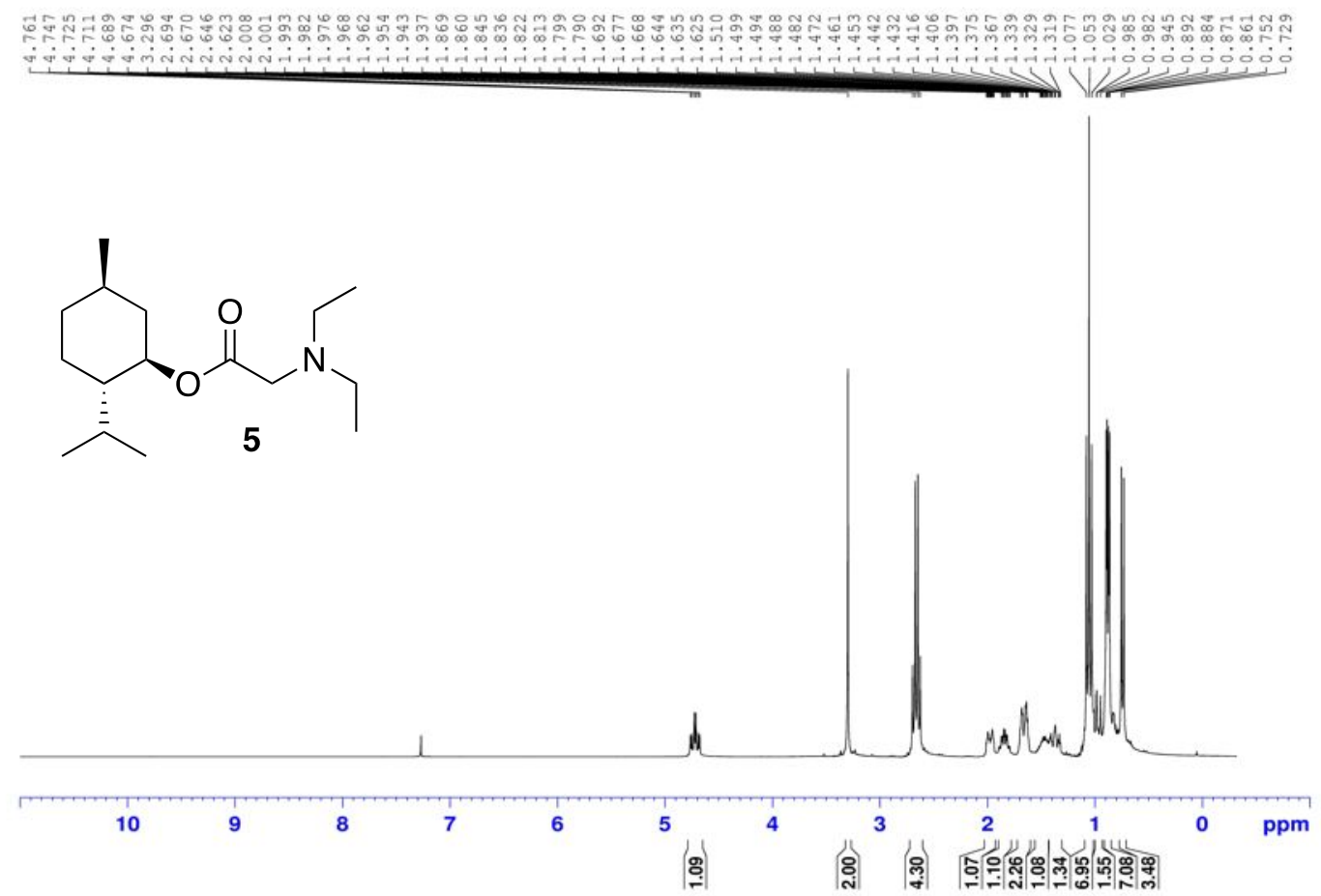

Figure S7. $1 \mathrm{H} \mathrm{NMR}$ of compound $\mathbf{5}\left(\mathrm{CDCl}_{3}, 300 \mathrm{MHz}\right)$.

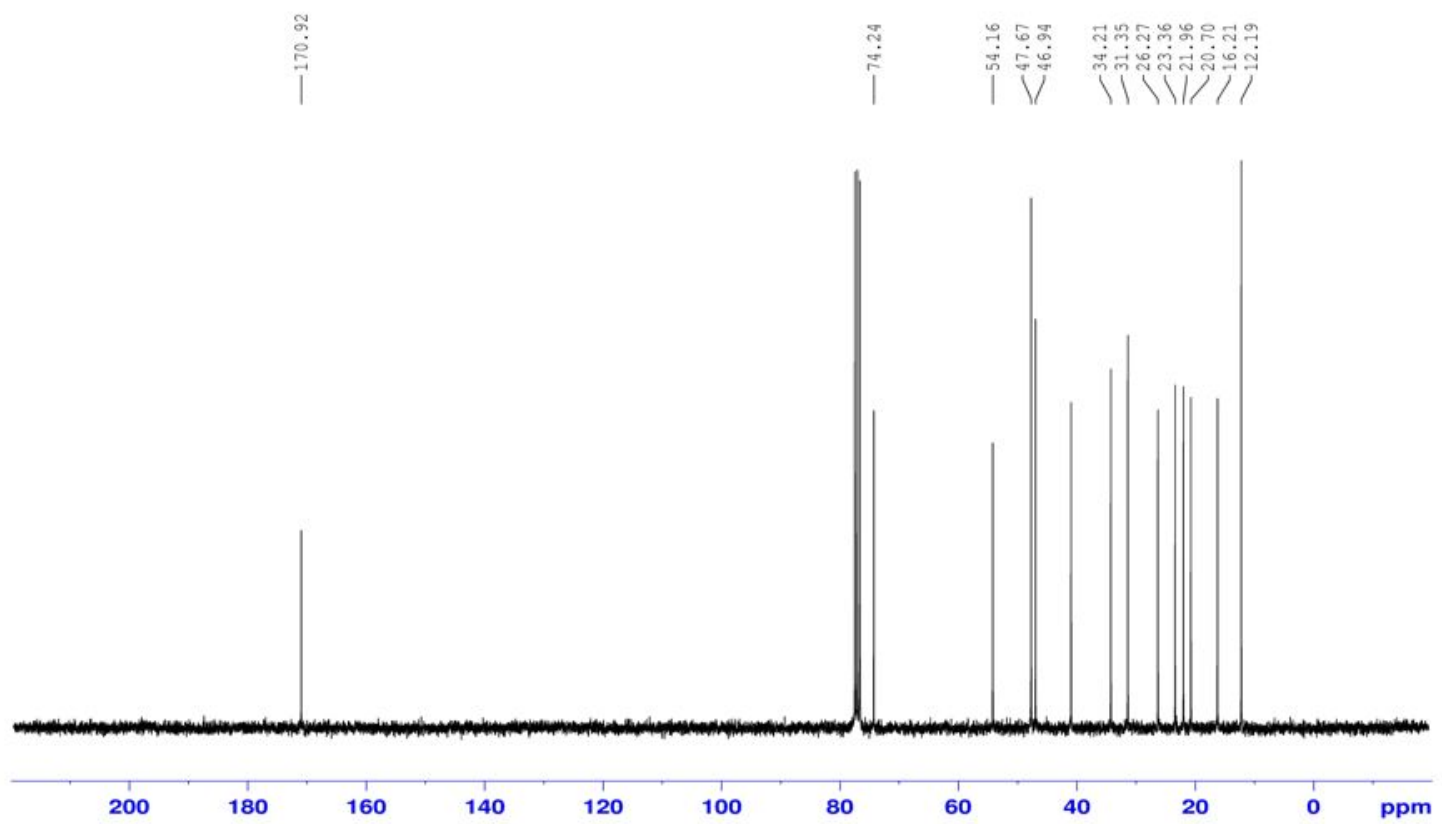

Figure S8. $13 \mathrm{C}$ NMR of compound $5\left(\mathrm{CDCl}_{3}, 75 \mathrm{MHz}\right)$. 


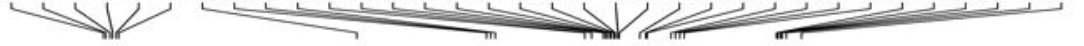<smiles>CCCN(CCC)CC(=O)OC1C[C@@H](C)CC[C@H]1C(C)C</smiles>

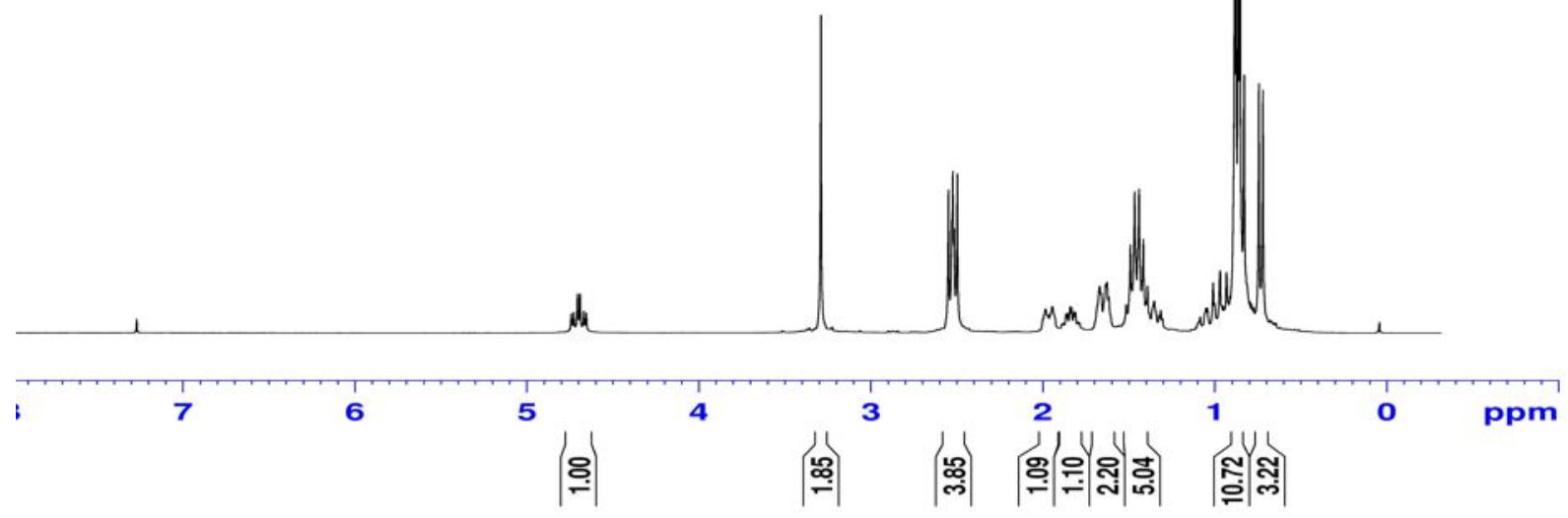

Figure S9. $1 \mathrm{H} \mathrm{NMR}$ of compound $6\left(\mathrm{CDCl}_{3}, 300 \mathrm{MHz}\right)$.

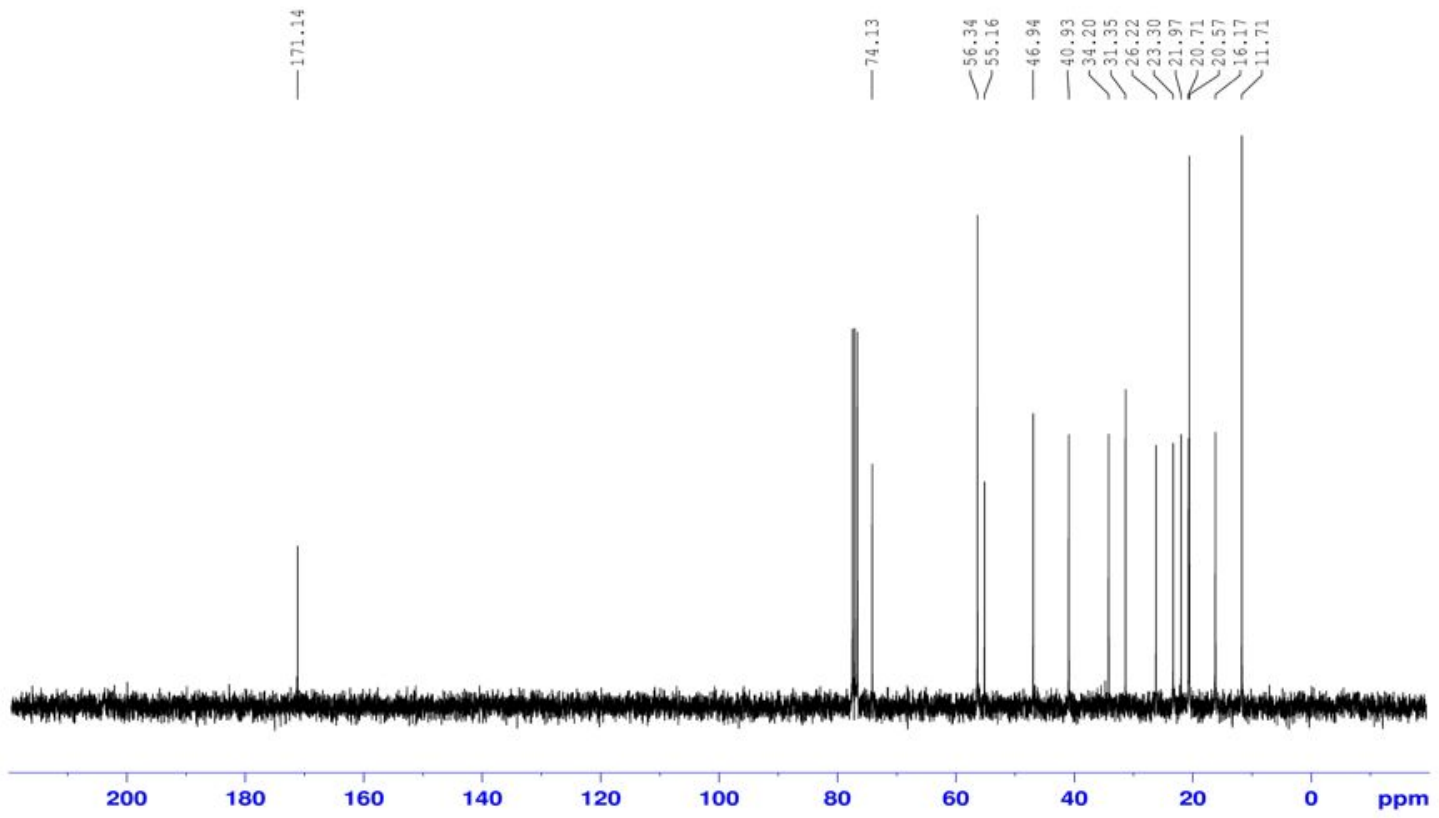

Figure S10. $13 \mathrm{C}$ NMR of compound $6\left(\mathrm{CDCl}_{3}, 75 \mathrm{MHz}\right)$. 


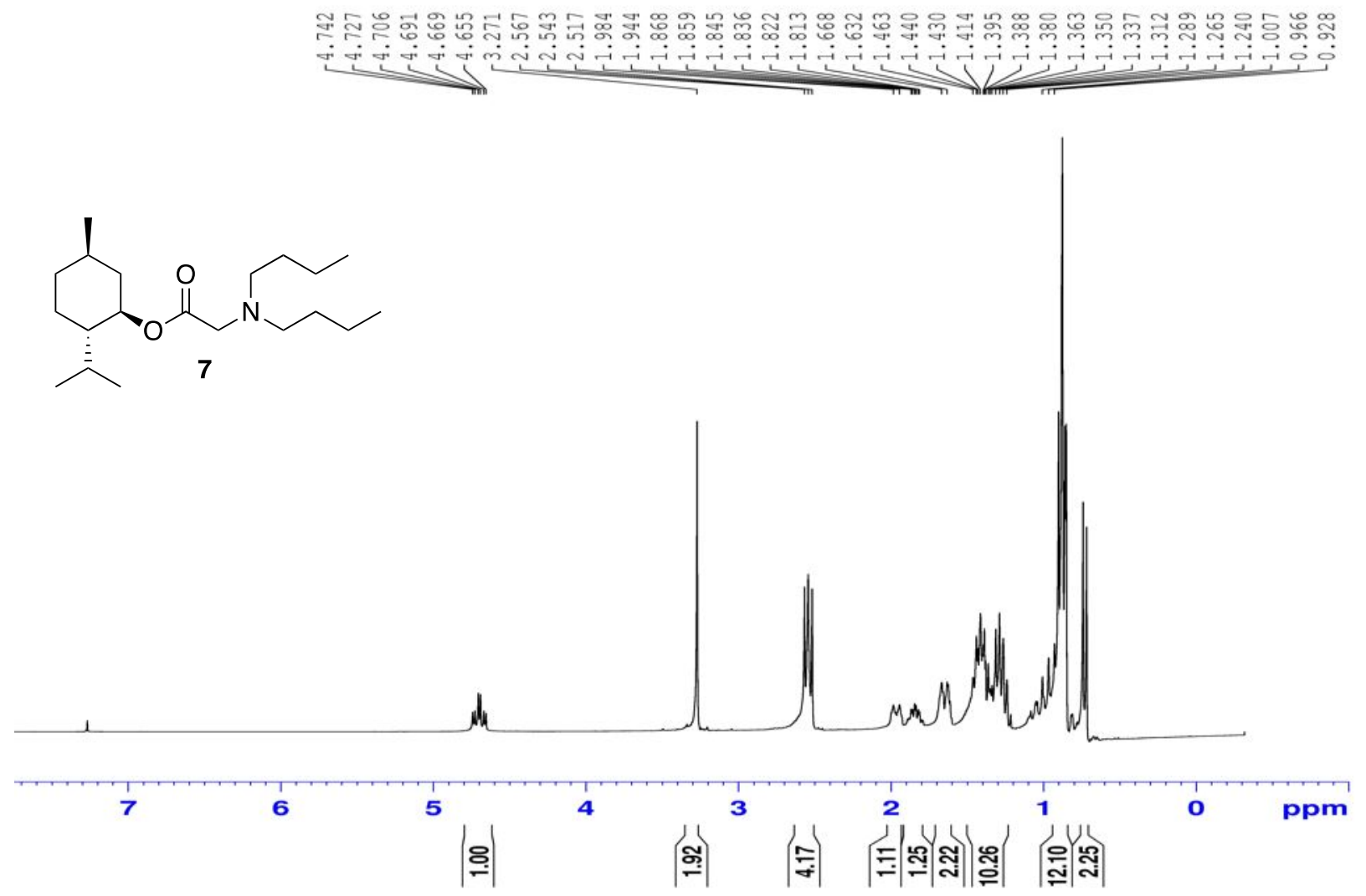

Figure S11. $1 \mathrm{H}$ NMR of compound $7\left(\mathrm{CDCl}_{3}, 300 \mathrm{MHz}\right)$.

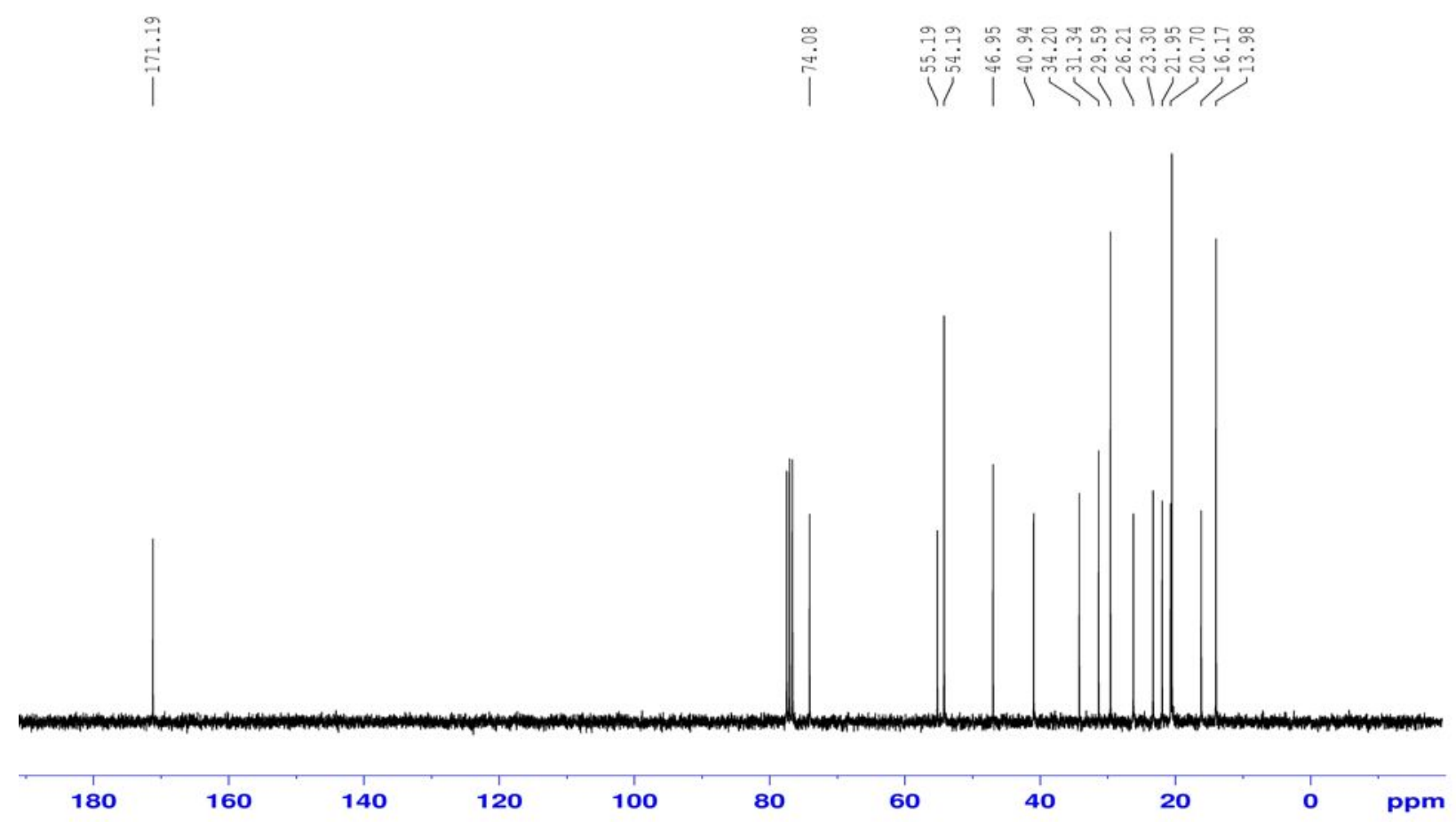

Figure S12. $13 \mathrm{C}$ NMR of compound $7\left(\mathrm{CDCl}_{3}, 75 \mathrm{MHz}\right)$. 


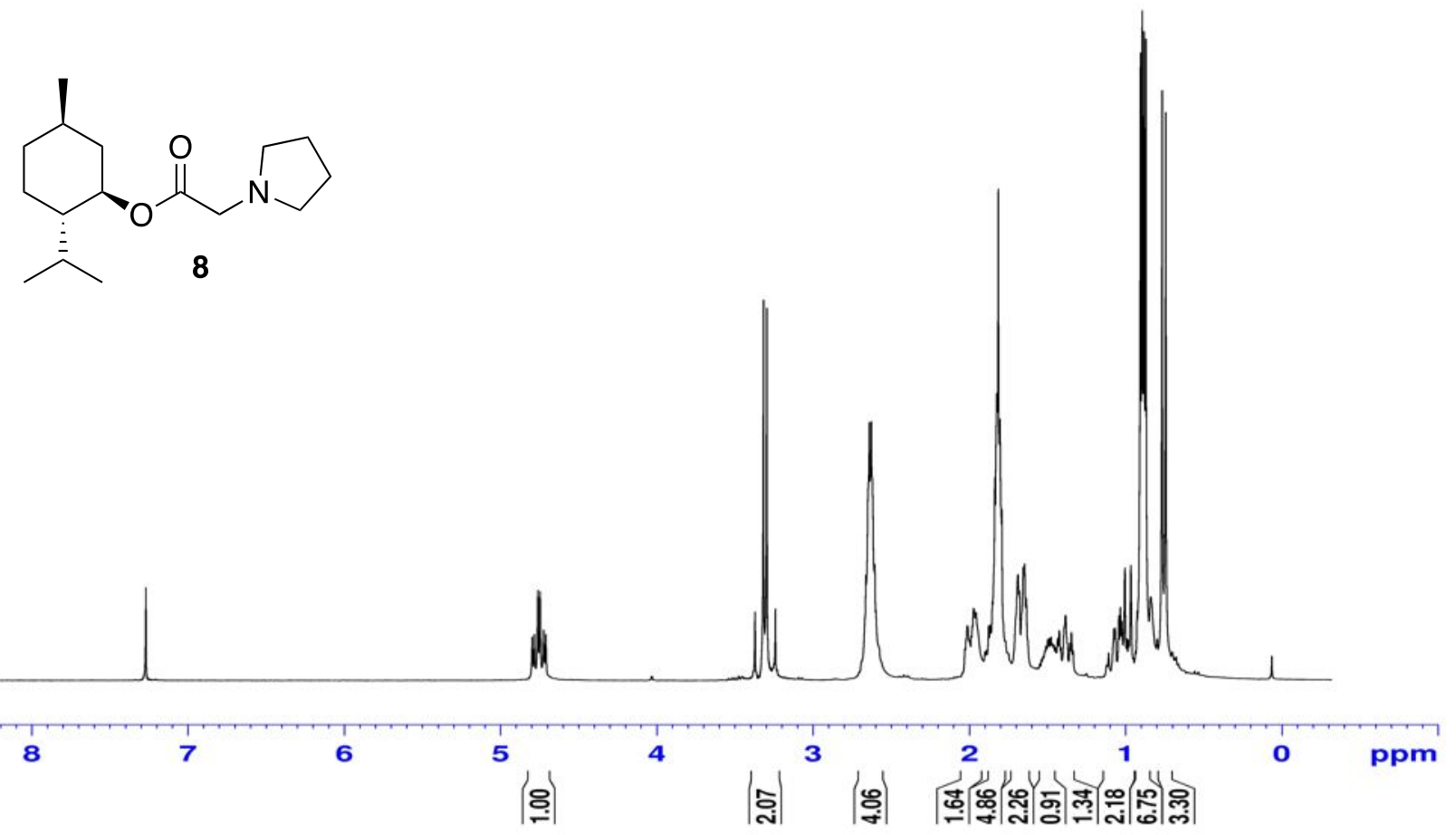

Figure S13. $1 \mathrm{H} \mathrm{NMR}$ of compound $\mathbf{8}\left(\mathrm{CDCl}_{3}, 300 \mathrm{MHz}\right)$.

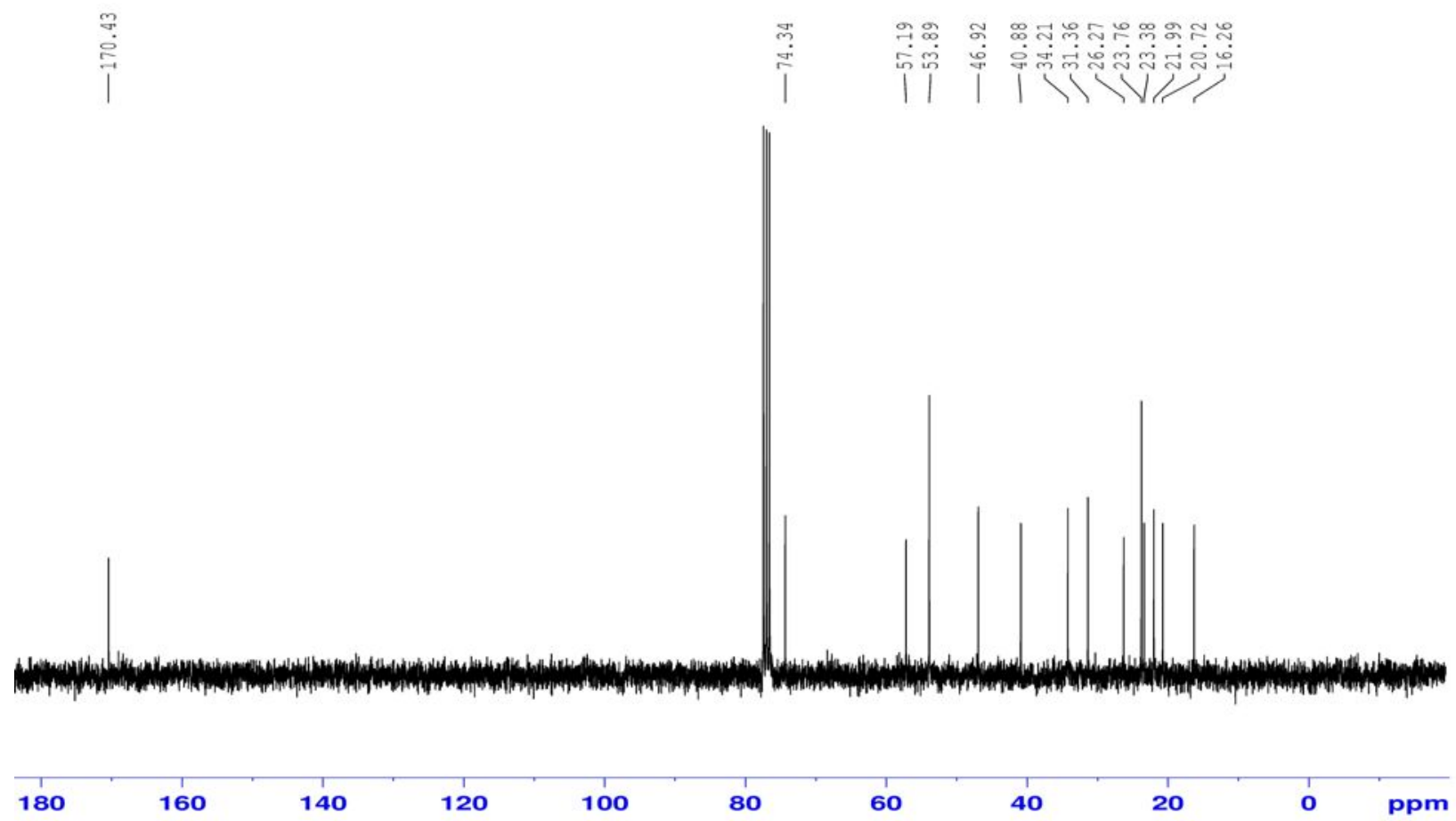

Figure S14. 13C NMR of compound $8\left(\mathrm{CDCl}_{3}, 75 \mathrm{MHz}\right)$. 


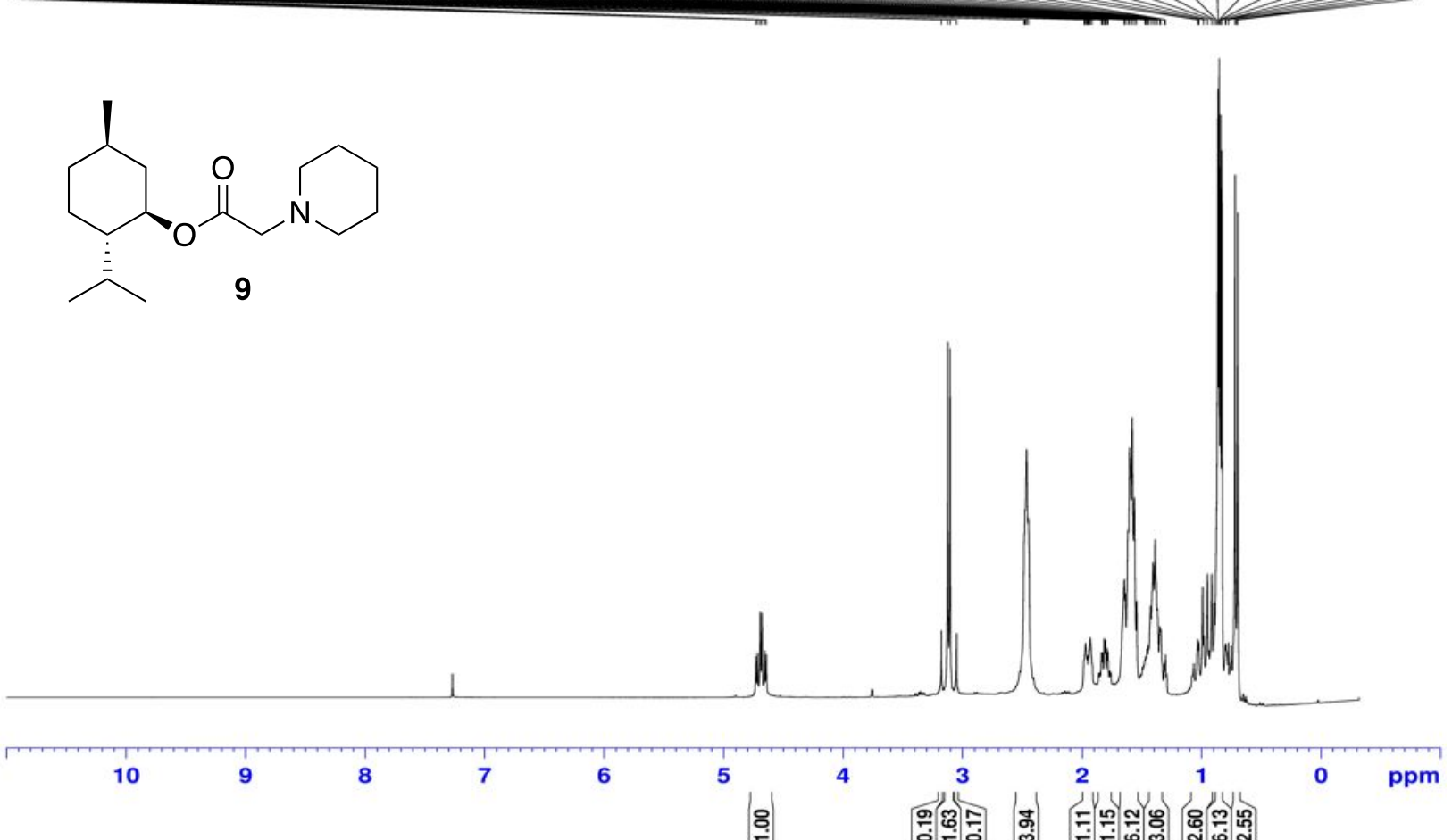

Figure S15. $1 \mathrm{H}$ NMR of compound $9\left(\mathrm{CDCl}_{3}, 300 \mathrm{MHz}\right)$.

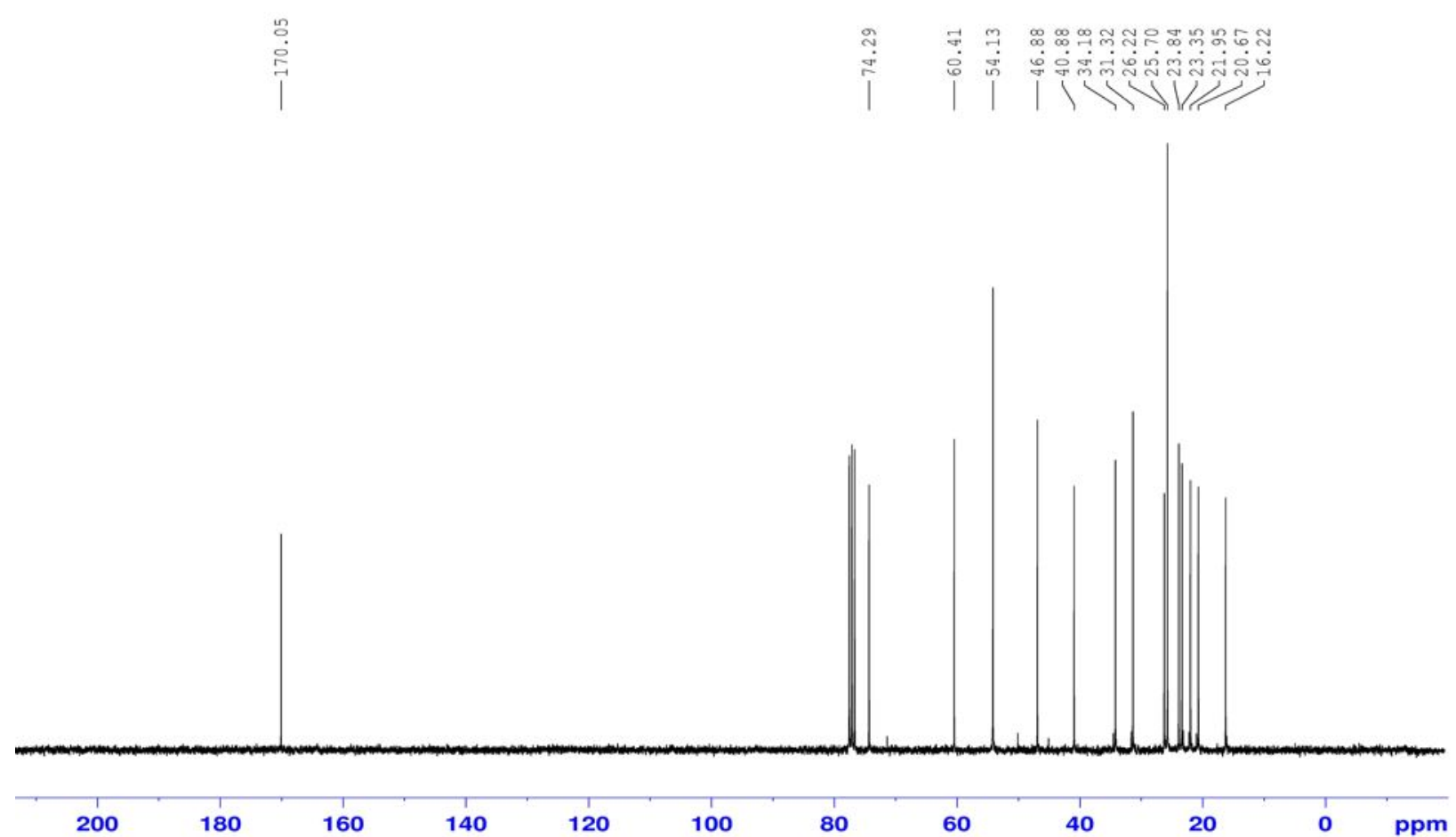

Figure S16. $1 \mathrm{H} \mathrm{NMR} \mathrm{of} \mathrm{compound} 9\left(\mathrm{CDCl}_{3}, 75 \mathrm{MHz}\right)$. 
<smiles>CC(C)[C@H]1CC[C@@H](C)C[C@H]1OC(=O)CNC1CC1</smiles>

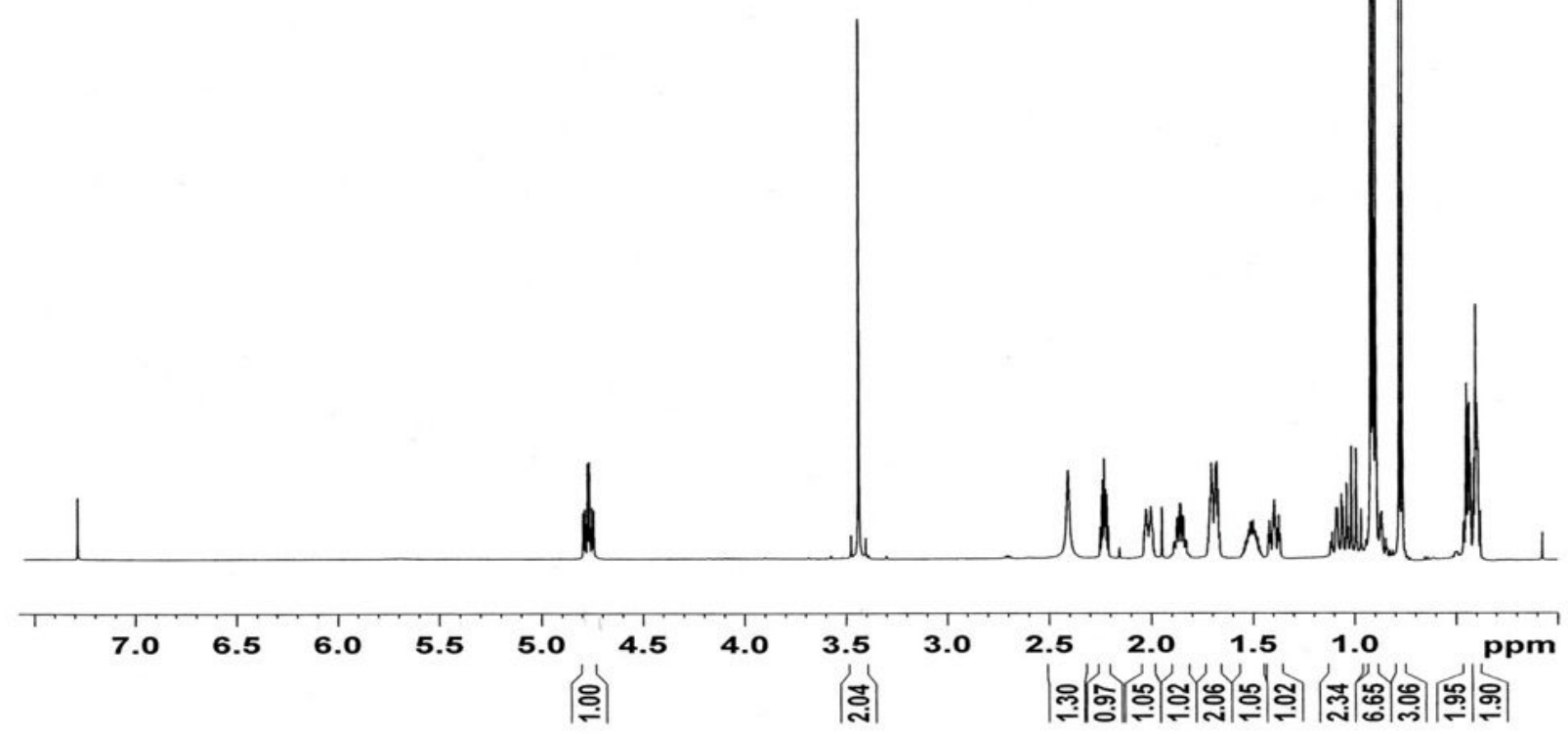

Figure S17. $1 \mathrm{H} \mathrm{NMR}$ of compound $10\left(\mathrm{CDCl}_{3}, 300 \mathrm{MHz}\right)$.

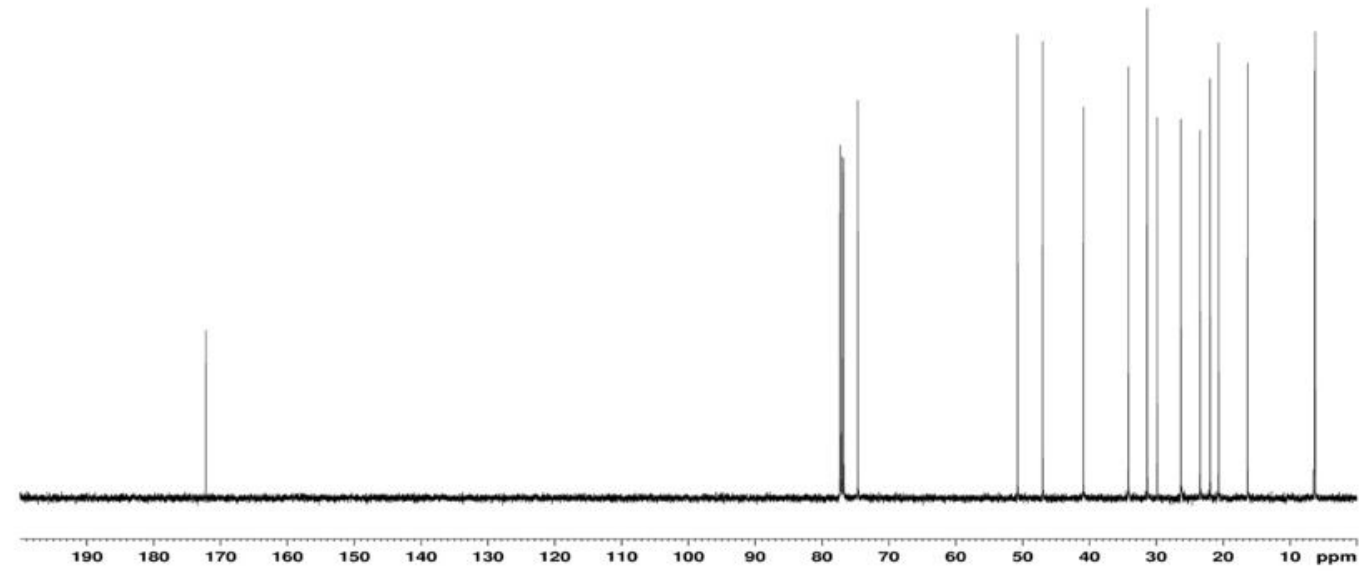

Figure S18. $13 \mathrm{C}$ NMR of compound $10\left(\mathrm{CDCl}_{3}, 75 \mathrm{MHz}\right)$. 

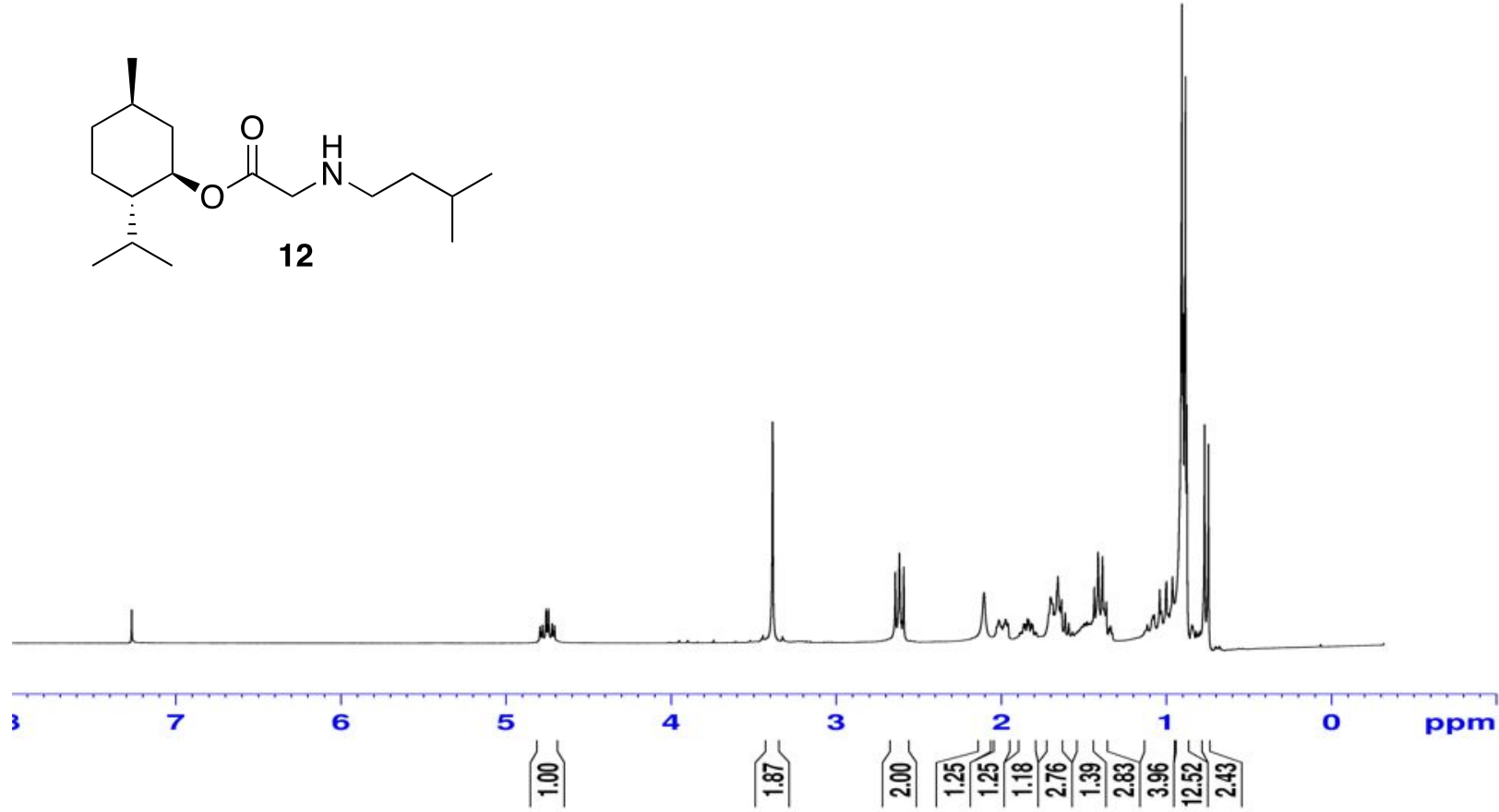

Figure S19. $1 \mathrm{H} \mathrm{NMR}$ of compound $12\left(\mathrm{CDCl}_{3}, 300 \mathrm{MHz}\right)$.

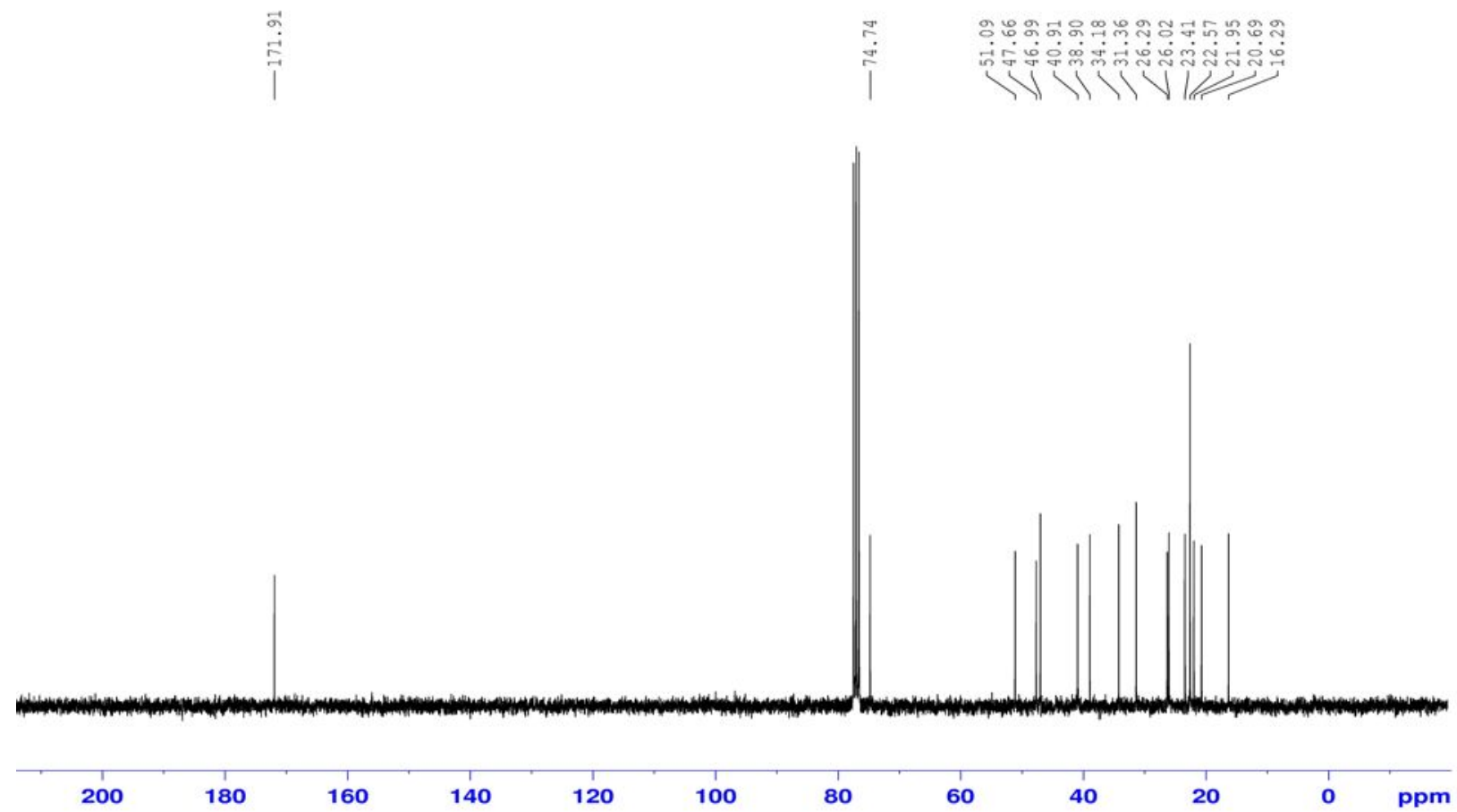

Figure S20. $1 \mathrm{H} \mathrm{NMR}$ of compound $12\left(\mathrm{CDCl}_{3}, 75 \mathrm{MHz}\right)$. 
<smiles>CC(C)[C@H]1CC[C@@H](C)C[C@H]1OC(=O)CN(C)Cc1ccccc1</smiles>

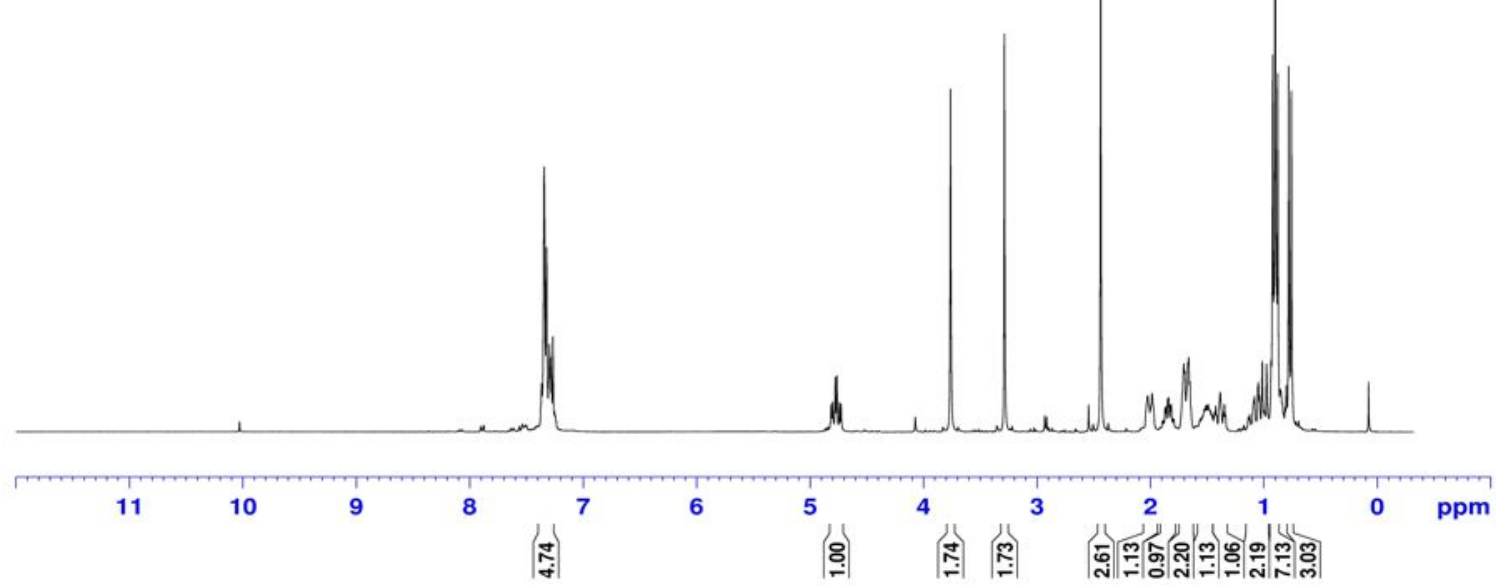

Figure S21. 1H NMR of compound $13\left(\mathrm{CDCl}_{3}, 300 \mathrm{MHz}\right)$.

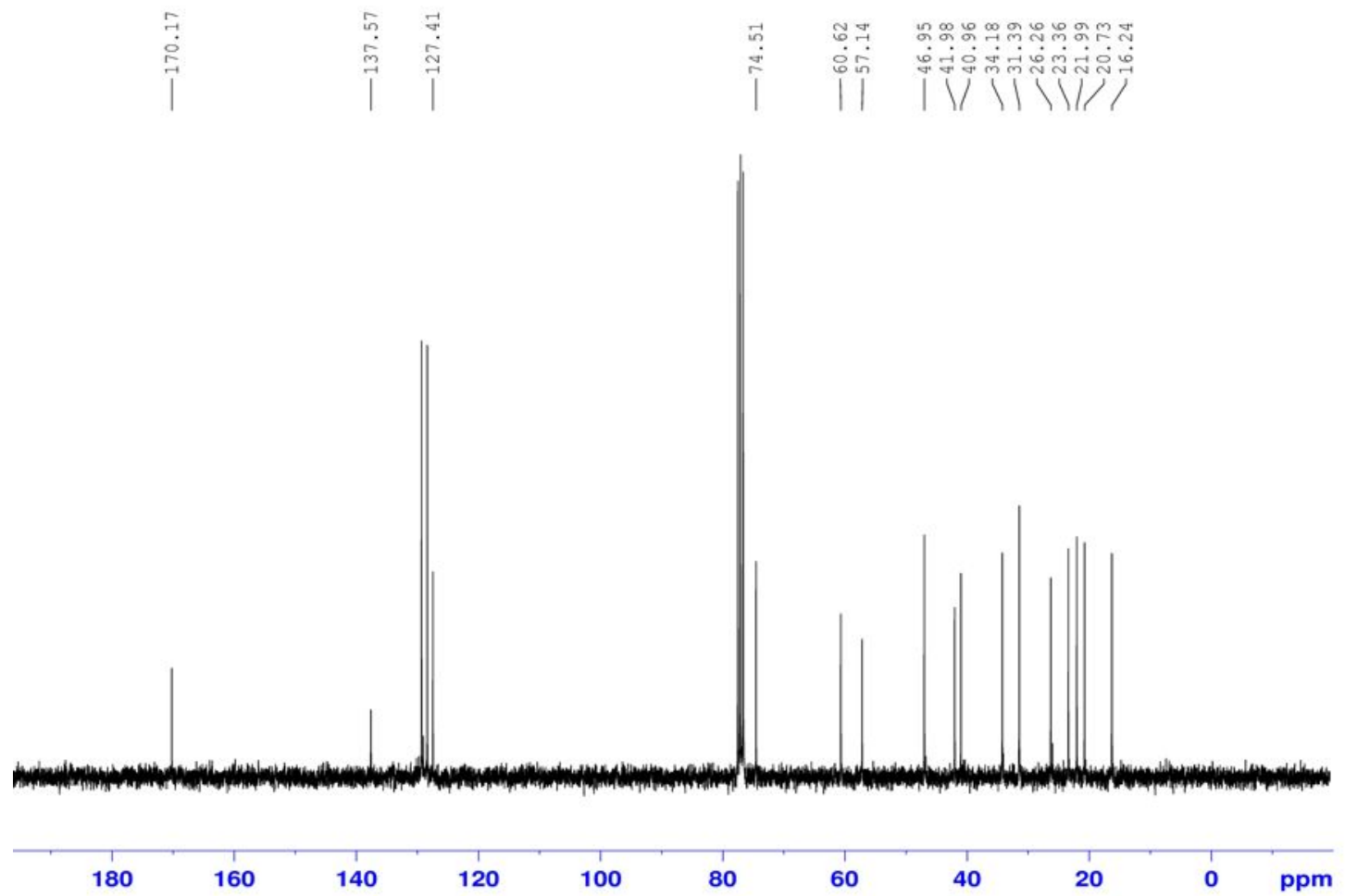

Figure S22. 13C NMR of compound $2\left(\mathrm{CDCl}_{3}, 75 \mathrm{MHz}\right)$. 


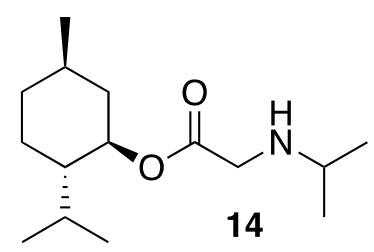

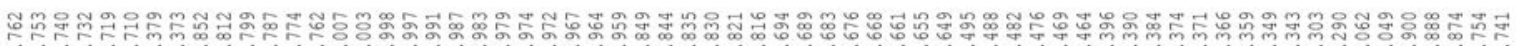

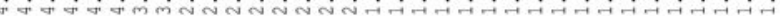

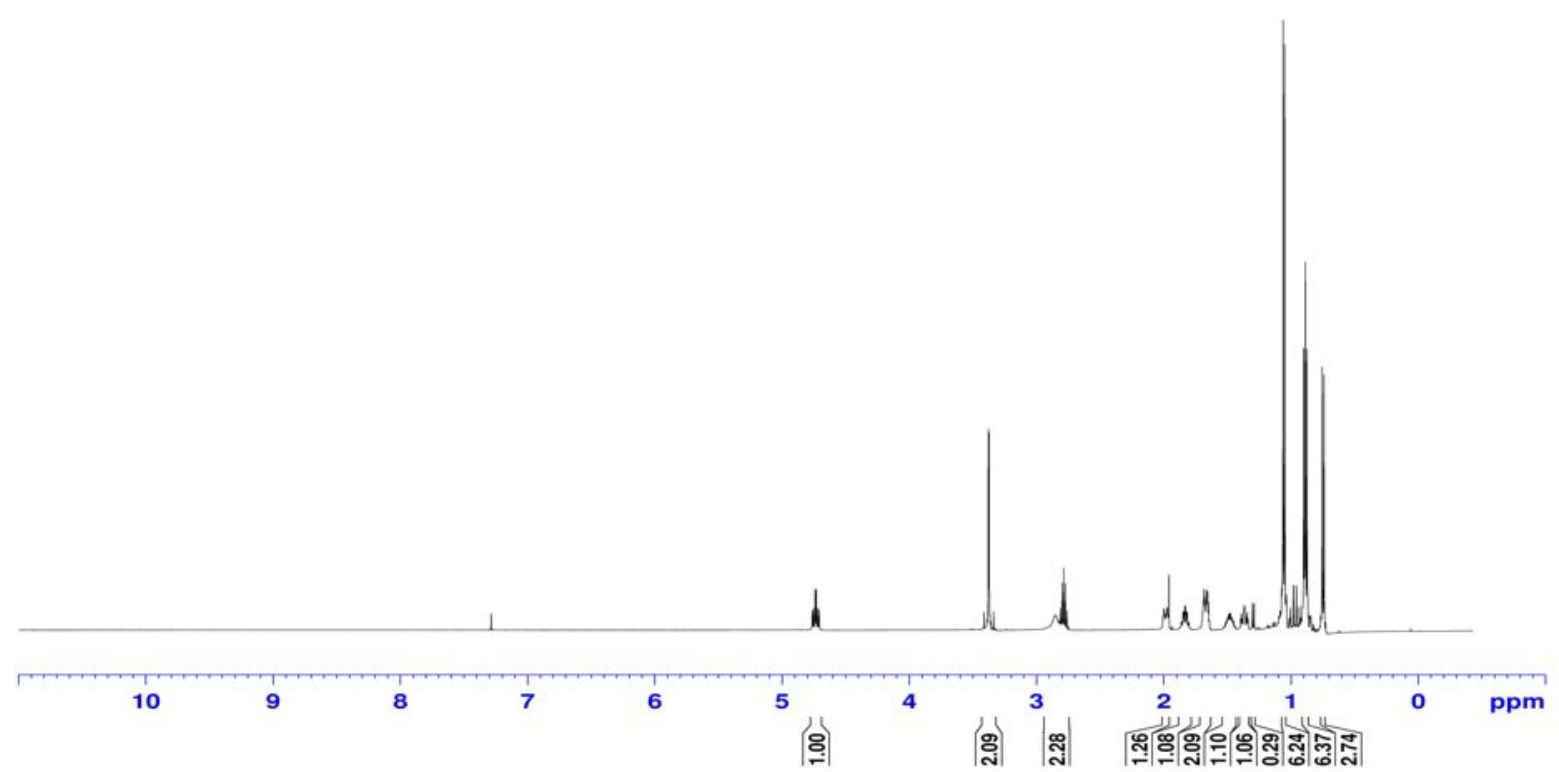

Figure S23. $1 \mathrm{H} \mathrm{NMR}$ of compound $14\left(\mathrm{CDCl}_{3}, 300 \mathrm{MHz}\right)$.

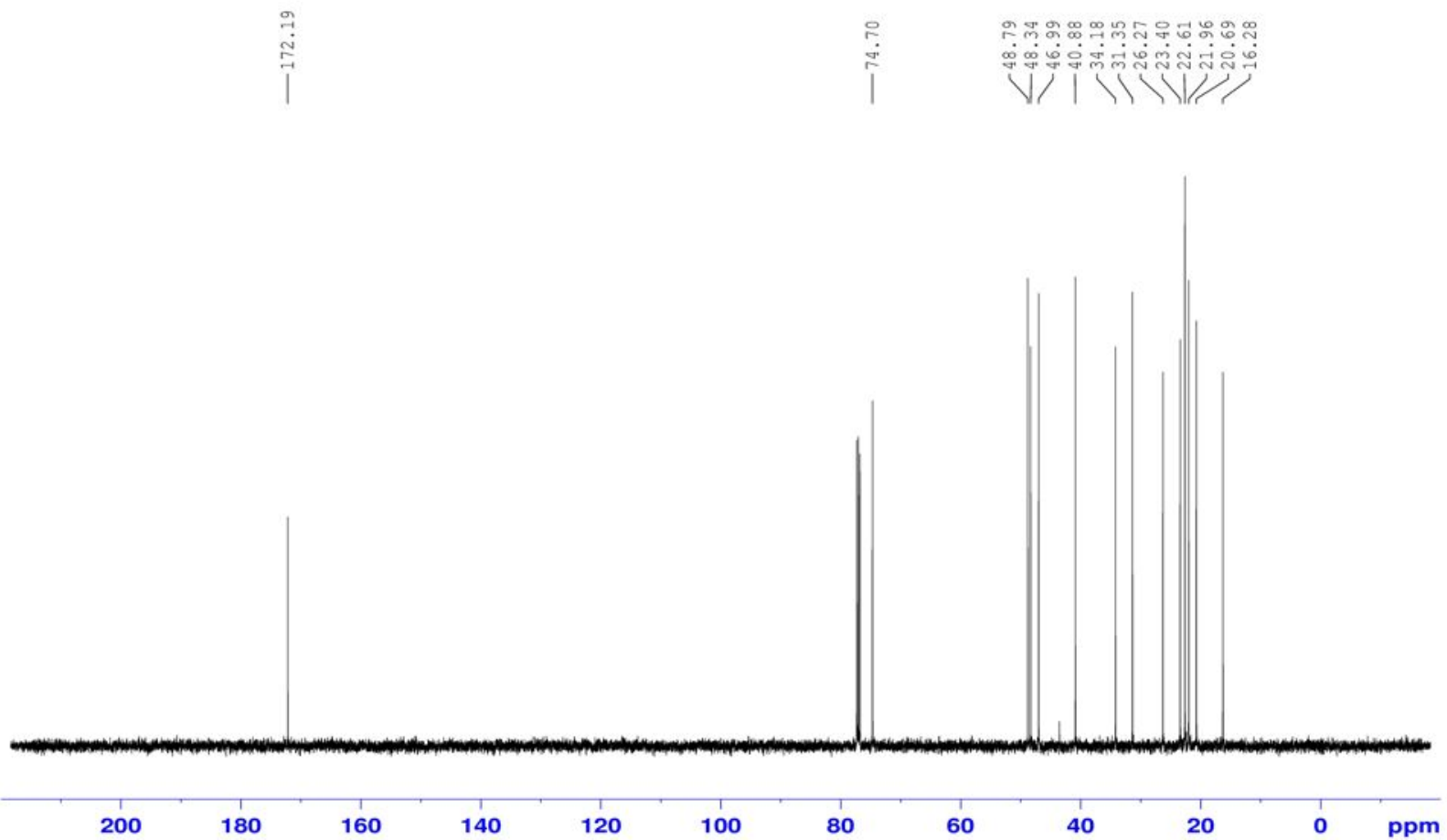

Figure S24. 13C NMR of compound $14\left(\mathrm{CDCl}_{3}, 75 \mathrm{MHz}\right)$. 
<smiles>CC(C)[C@@H]1CC[C@@H](C)C[C@@H]1OC([13CH3])=O</smiles>

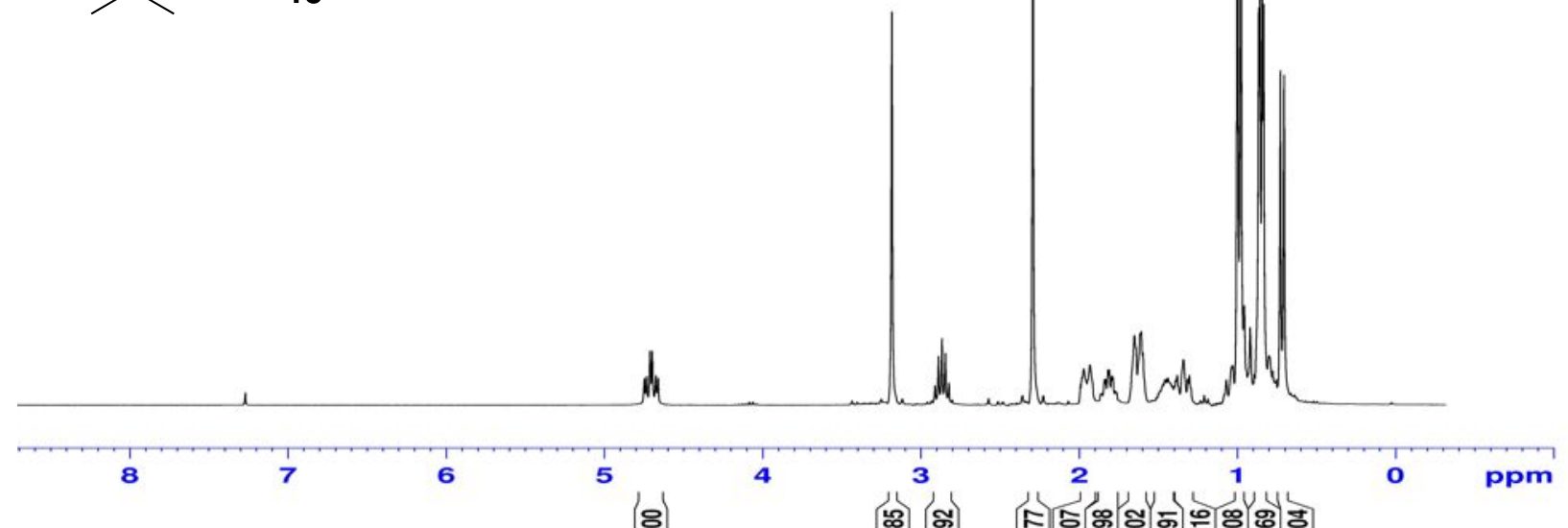

().

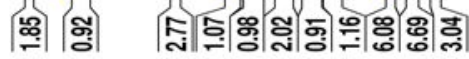

Figure S25. $1 \mathrm{H} \mathrm{NMR}$ of compound $15\left(\mathrm{CDCl}_{3}, 300 \mathrm{MHz}\right)$.

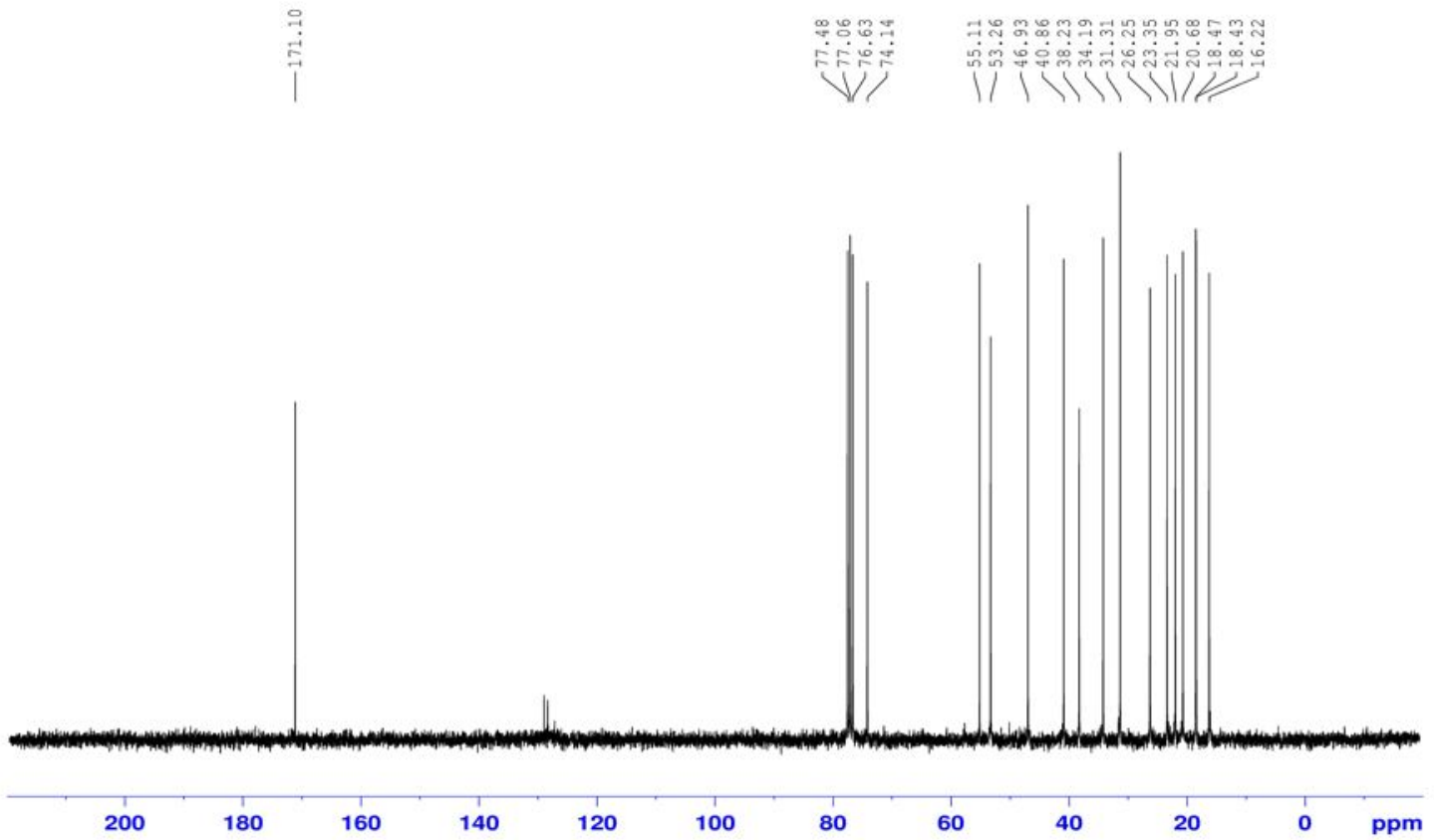

Figure S26. $13 \mathrm{C}$ NMR of compound $15\left(\mathrm{CDCl}_{3}, 75 \mathrm{MHz}\right)$. 
<smiles>CC(C)CN(CC(=O)OC1CC(C)CCC1C(C)C)CC(C)C</smiles>

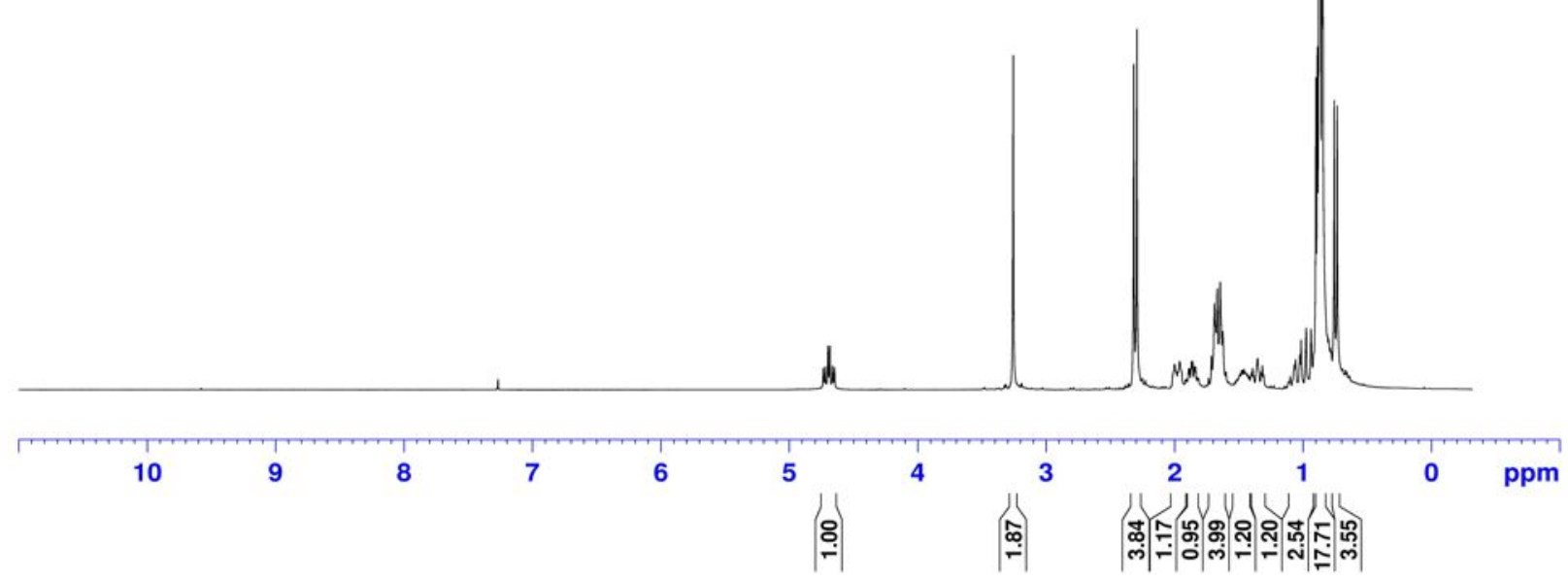

Figure S27. $1 \mathrm{H} \mathrm{NMR}$ of compound $16\left(\mathrm{CDCl}_{3}, 300 \mathrm{MHz}\right)$.

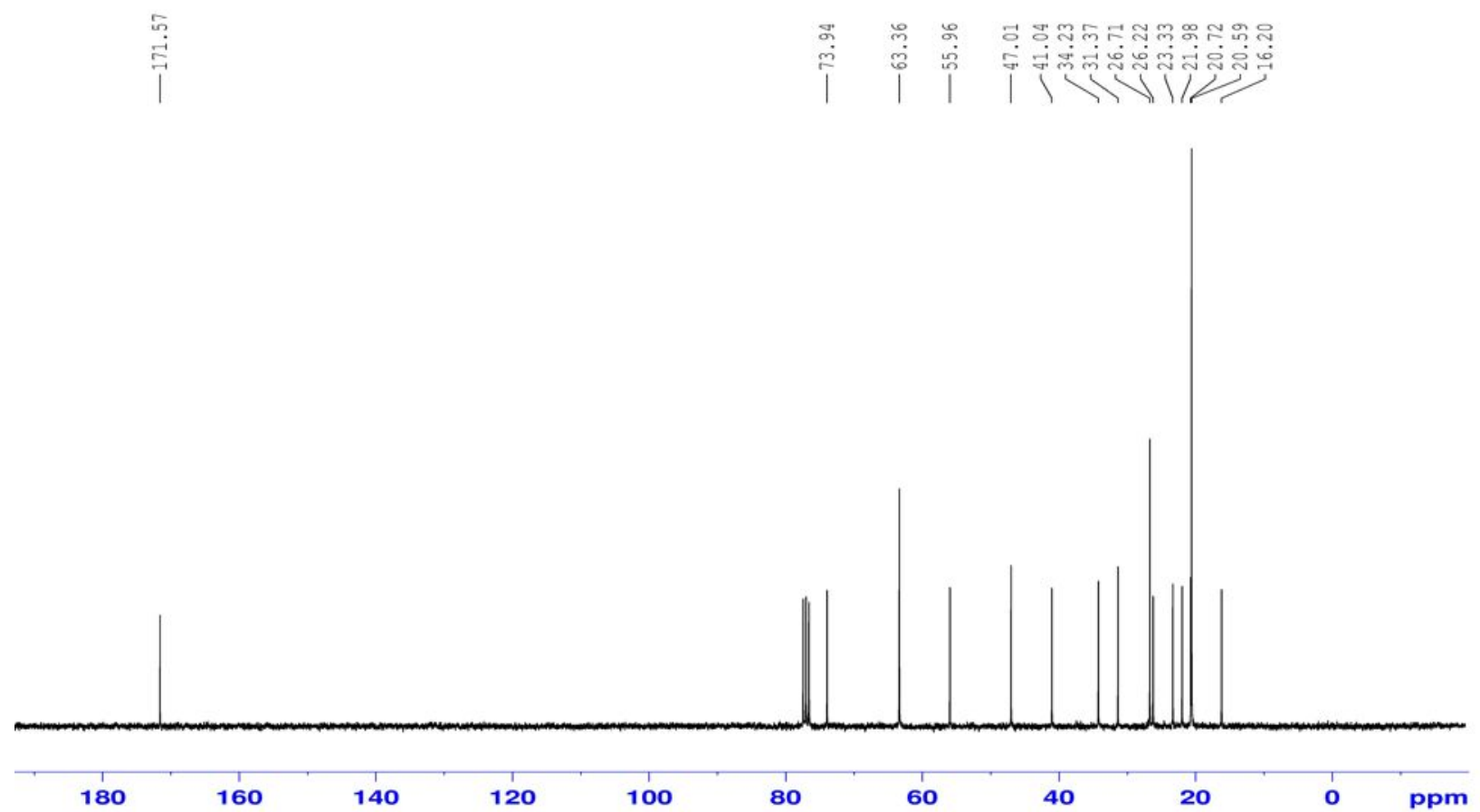

Figure S28. $13 \mathrm{C}$ NMR of compound $16\left(\mathrm{CDCl}_{3}, 75 \mathrm{MHz}\right)$. 

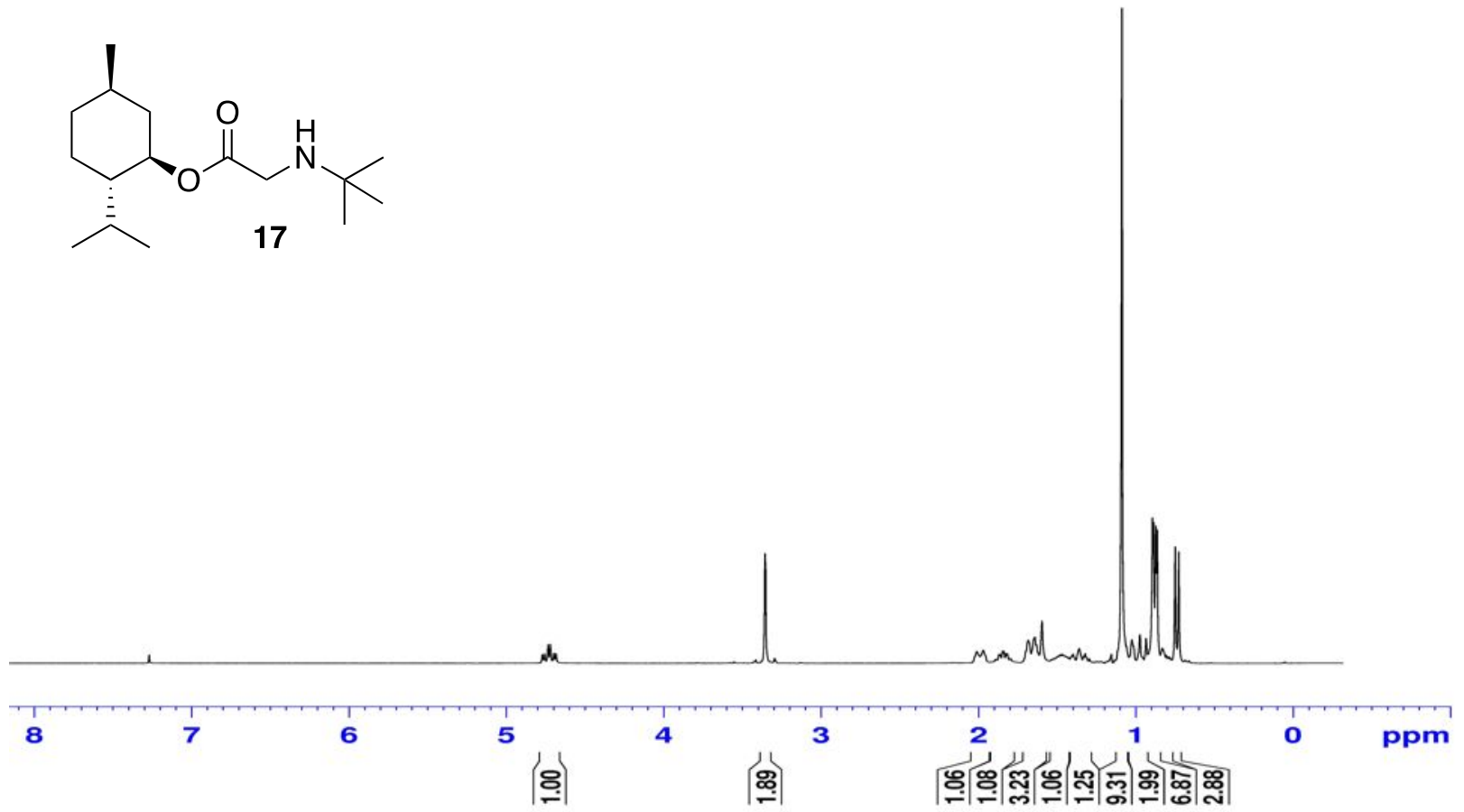

Figure S29. $1 \mathrm{H}$ NMR of compound $17\left(\mathrm{CDCl}_{3}, 300 \mathrm{MHz}\right)$.

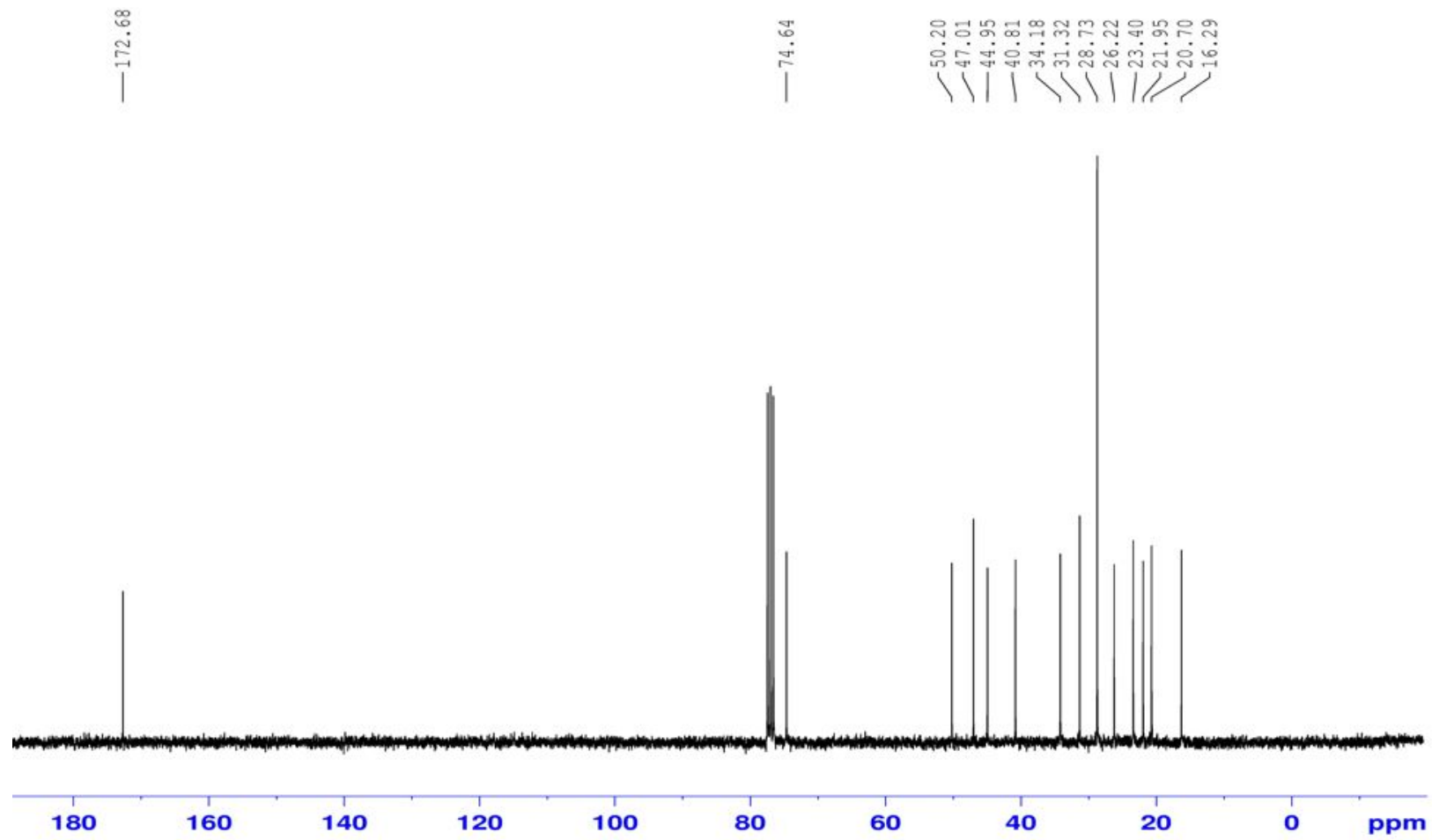

Figure S30. 13C NMR of compound $17\left(\mathrm{CDCl}_{3}, 75 \mathrm{MHz}\right)$. 
<smiles>CC(C)[C@H]1CC[C@@H](C)C[C@H]1OC(=O)CN(C)CCc1ccccn1</smiles>

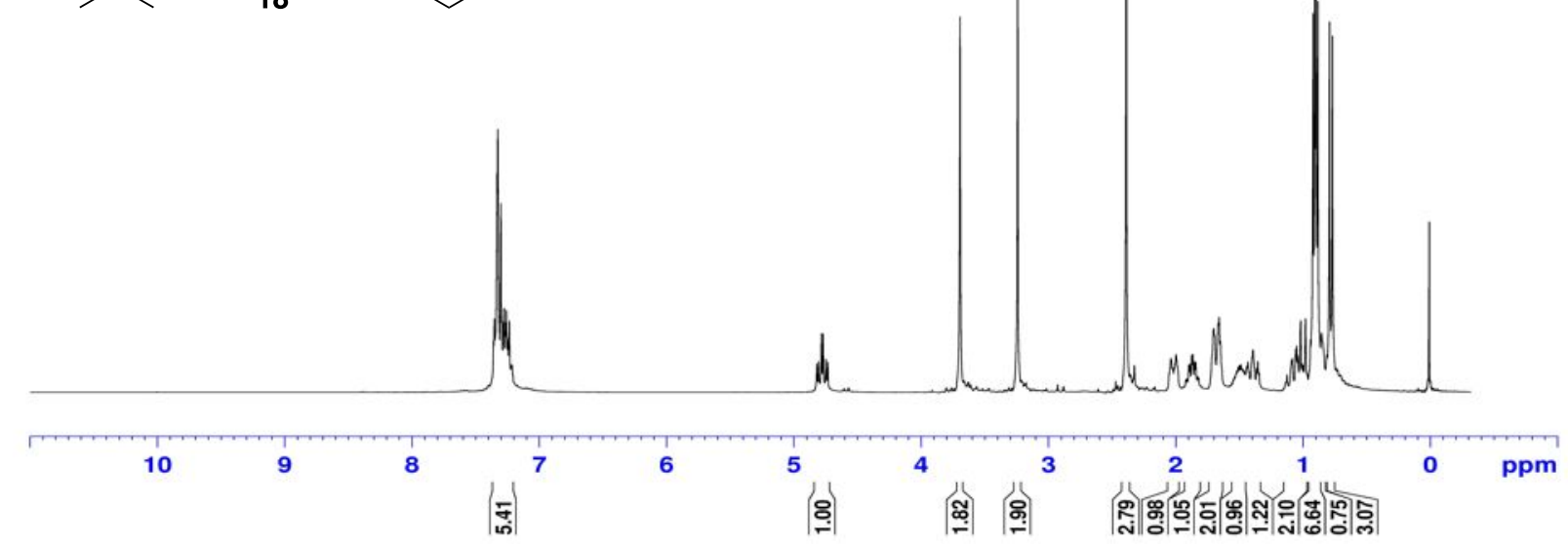

Figure S31. 1H NMR of compound $18\left(\mathrm{CDCl}_{3}, 300 \mathrm{MHz}\right)$.

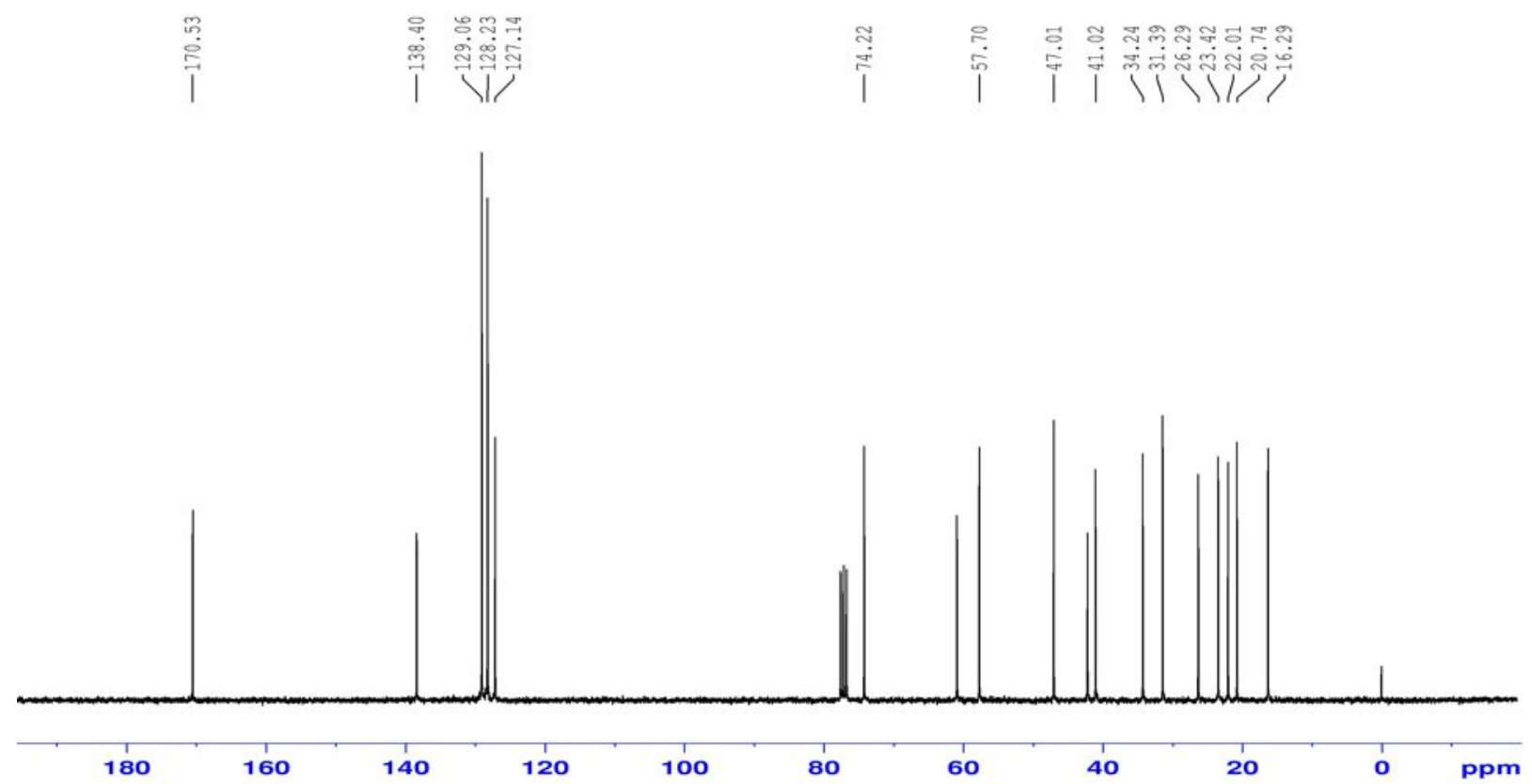

Figure S32. $13 \mathrm{C}$ NMR of compound $18\left(\mathrm{CDCl}_{3}, 75 \mathrm{MHz}\right)$. 
<smiles>CC1CC[C@@H](C(C)C)C(OC(=O)CN(CC(=O)O[C@H]2C[C@H](C)CC[C@H]2C(C)C)C(C)C)C1</smiles>

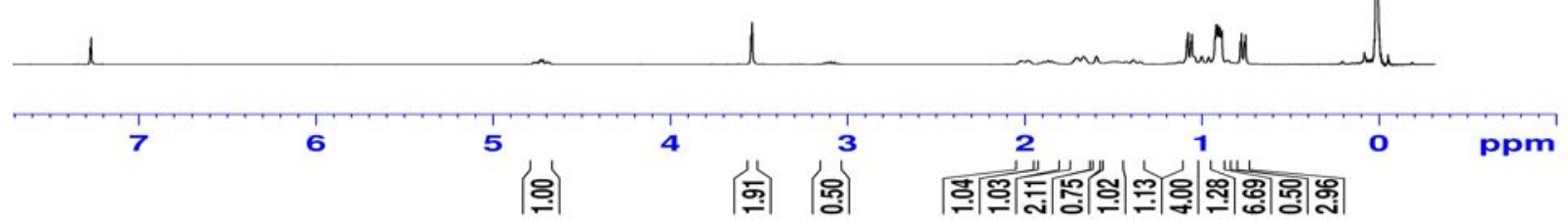

Figure S33. $1 \mathrm{H} \mathrm{NMR}$ of compound $20\left(\mathrm{CDCl}_{3}, 300 \mathrm{MHz}\right)$.

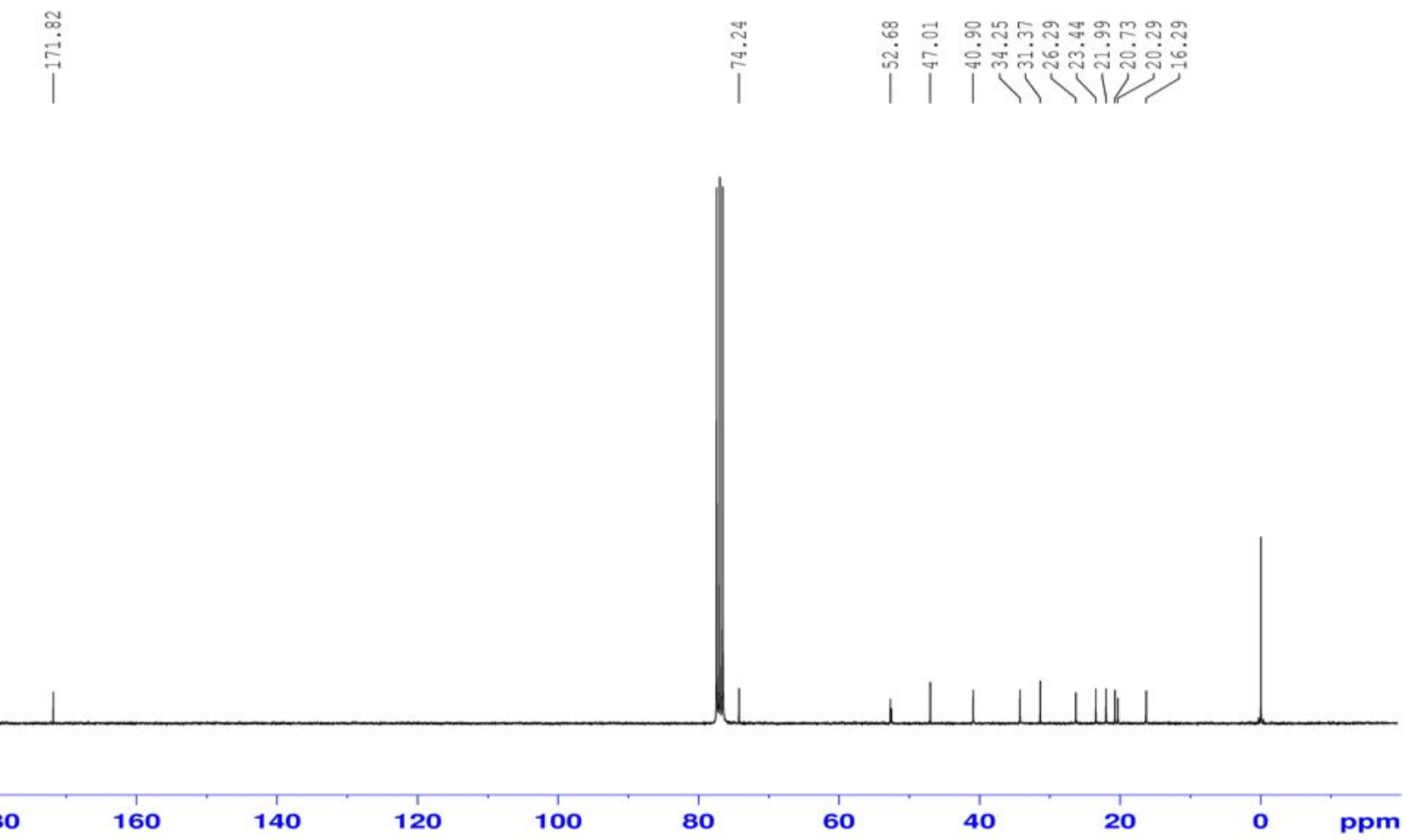

Figure S34. 13C NMR of compound $20\left(\mathrm{CDCl}_{3}, 75 \mathrm{MHz}\right)$. 


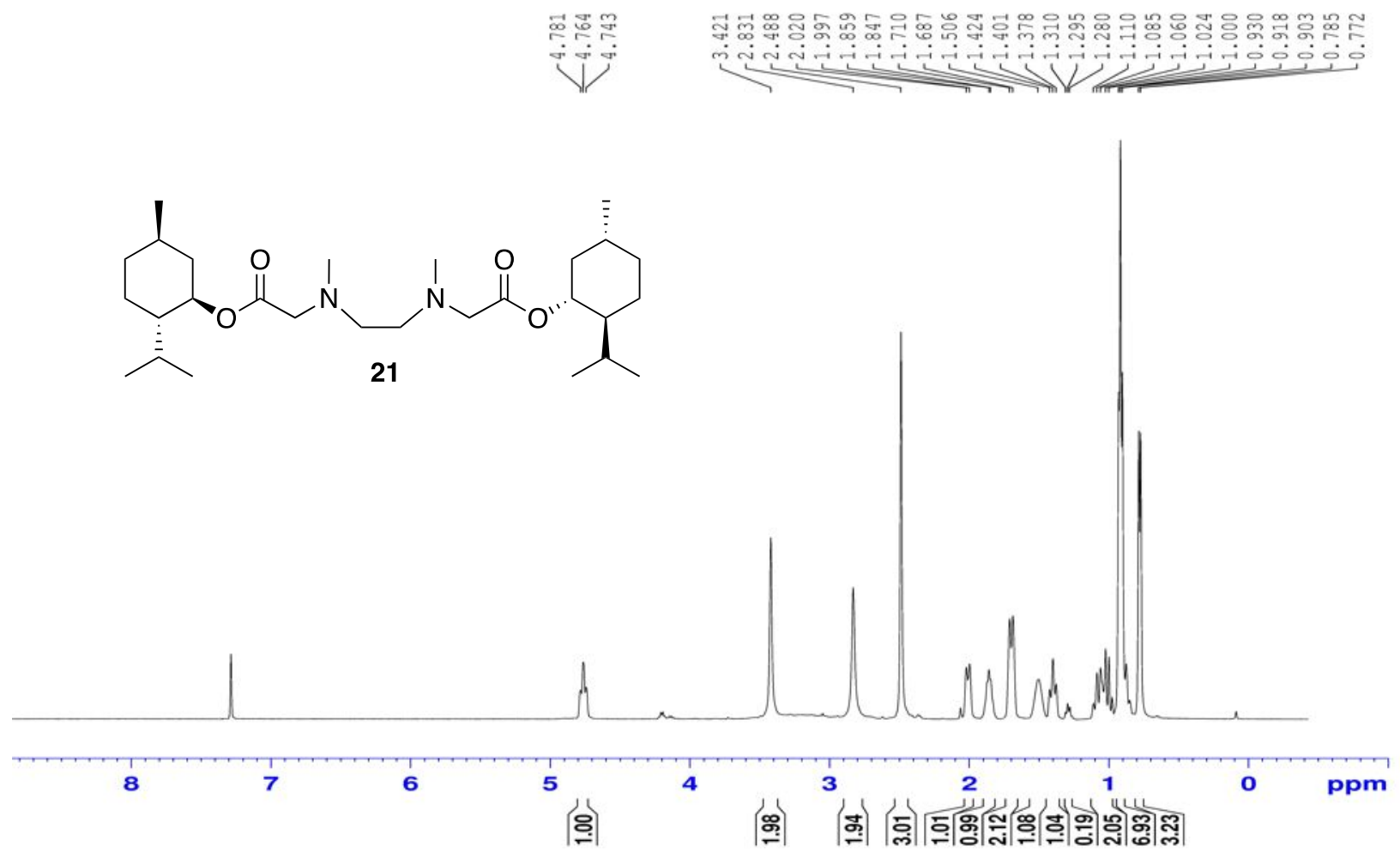

Figure S35. $1 \mathrm{H} \mathrm{NMR}$ of compound $21\left(\mathrm{CDCl}_{3}, 300 \mathrm{MHz}\right)$.

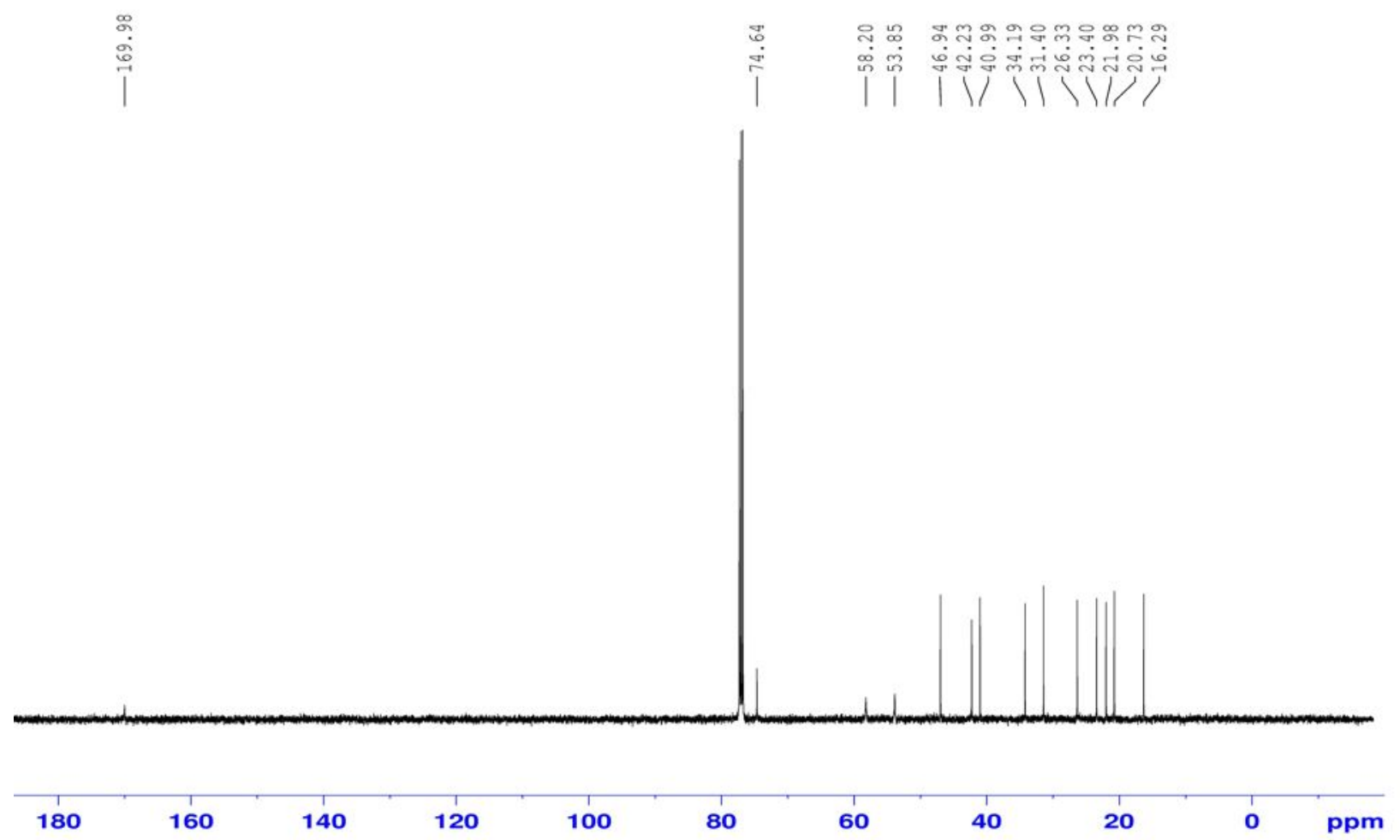

Figure S36. 13C NMR of compound $21\left(\mathrm{CDCl}_{3}, 75 \mathrm{MHz}\right)$. 